\title{
Stream temperature evolution in Switzerland over the last 50 years Supplementary Materials
}

\author{
Adrien Michel ${ }^{1,2}$, Tristan Brauchli ${ }^{1,3}$, Michael Lehning ${ }^{1,2}$, Bettina Schaefli ${ }^{3}$, and Hendrik Huwald ${ }^{1,2}$ \\ ${ }^{1}$ School of Architecture, Civil and Environmental Engineering, École Polytechnique Fédérale de Lausanne (EPFL), \\ Switzerland \\ ${ }^{2}$ WSL Institute for Snow and Avalanche Research (SLF), Davos, Switzerland \\ ${ }^{3}$ Faculty of Geosciences and Environment, University of Lausanne, Lausanne, Switzerland
}

Correspondence: A. Michel (adrien.michel@epfl.ch)

\begin{abstract}
The present supplementary material is complementing the key elements of the study presented in the main part of this work. It either expands on results which were too voluminous for the main article or gives elements for better understanding and potential reproduction of the results. Often, the article only shows pertinent examples while the larger body of corresponding results is included here. The first part is a collection of additional tables and figures related to the data and

5 methods presented (Section S1), followed by more figures, detailing and complementing the main results in the article (Section S2). All descriptions and explanations necessary to understand the material below, as well as all the general conclusions, are provided in the text of the paper. The availability of the data and code are also mentioned and discussed in the article.
\end{abstract}

\section{S1 Data and methods supplementary materials}

\section{S1.1 Excluded catchments}

Some catchments have been excluded from the analysis for various reasons. They are listed below in Table S1.

Table S1. River measurement stations removed from the study and justifications. The hydrological regimes are Swiss Plateau and Jura regime (SPJ), Alpine regime (ALP), Downstream lake regime (DLA), and Regime strongly influenced by hydropeaking (HYP). The data providers are the Swiss Federal Office for the Environment (FOEN), the Office for water and waste of the Canton of Bern (AWA) and the Office for waste, water, energy and air of the Canton Zurich (AWEL).

\begin{tabular}{lccccc}
\hline River & $\begin{array}{c}\text { Temperature } \\
\text { measurement }\end{array}$ & $\begin{array}{c}\text { Discharge } \\
\text { measurement }\end{array}$ & $\begin{array}{c}\text { Hydrological } \\
\text { regime }\end{array}$ & $\begin{array}{c}\text { Data } \\
\text { provider }\end{array}$ & $\begin{array}{l}\text { Reason for } \\
\text { removal }\end{array}$ \\
\hline Foul in Moutier & $1995-2018$ & $1995-2018$ & SPJ & AWA & Issues in temperature values (no annual cycle) \\
Birse in Court, Pont de la STEP & $1996-2018$ & $1996-2018$ & SPJ & AWA & Below a water-treatment plant \\
Louibach in Gstaad, Badweidli & $1995-2018$ & $1994-2018$ & ALP & AWA & 1.5 year gap in water temperature, temperature only every 2 hours before 2000 \\
Suze in Péry, Vigier Ciment & $1996-2018$ & $1992-2018$ & SPJ & AWA & Disturbance because of a cement factory \\
Urtenen in Kernenried & $1997-2018$ & $1997-2018$ & SPJ & AWA & Below a water-treatment plant \\
Chalière in Moutier, Pont de la STEP & $1997-2018$ & $1997-2018$ & SPJ & AWA & 1.5 year gap in water temperature \\
Entschlige in Frutigen, Tropenhaus & $1998-2018$ & $1998-2018$ & ALP & AWA & Multiple gaps in the time series \\
Rhein in Weil, Palmrainbrücke & $1995-2018$ & $1992-2018$ & DLA & FOEN & Data only since 1995, already many sations on the Rhein \\
\hline
\end{tabular}


S1.2 MeteoSwiss stations details

Table S2 summarizes information about the MeteoSwiss stations used.

Table S2. Details of MeteoSwiss stations used including the periods for air temperature, precipitation, homogeneous air temperature and homogeneous precipitation time series.

\begin{tabular}{|c|c|c|c|c|c|c|c|c|}
\hline $\begin{array}{l}\text { Station } \\
\text { Name }\end{array}$ & $\begin{array}{l}\text { Station } \\
\text { Abbreviation }\end{array}$ & $\begin{array}{c}\text { Station } \\
\text { Elevation }\end{array}$ & $\begin{array}{c}\text { Easting } \\
\text { Coordinates }\end{array}$ & $\begin{array}{c}\text { Northing } \\
\text { Coordinates }\end{array}$ & $\begin{array}{c}\text { Air temp. } \\
\text { measurement }\end{array}$ & $\begin{array}{c}\text { Hom. air temp. } \\
\text { measurement }\end{array}$ & $\begin{array}{l}\text { Precipitation } \\
\text { measurement }\end{array}$ & $\begin{array}{c}\text { Hom. precipitation } \\
\text { measurement }\end{array}$ \\
\hline Adelboden & $\mathrm{ABO}$ & 609350 & 149001 & 1322 & $1959-2018$ & - & $1950-2018$ & - \\
\hline Altdorf & ALT & 690180 & 193564 & 438 & $1950-2018$ & $1950-2018$ & $1950-2018$ & $1950-2018$ \\
\hline Basel-Binningen & BAS & 610908 & 265611 & 316 & $1950-2018$ & $1950-2018$ & $1950-2018$ & $1950-2018$ \\
\hline Passo-del-Bernina & BEH & 798422 & 143020 & 2260 & $1972-2018$ & - & $1950-2018$ & - \\
\hline Bern-Zollikofen & BER & 601933 & 204409 & 552 & $1950-2018$ & $1950-2018$ & $1950-2018$ & $1950-2018$ \\
\hline La-Chaux-de-Fonds & $\mathrm{CDF}$ & 550919 & 214861 & 1017 & $1950-2018$ & $1950-2018$ & $1950-2018$ & $1950-2018$ \\
\hline Chasseral & $\mathrm{CHA}$ & 570845 & 220157 & 1599 & $1981-2018$ & - & 1981-2018 & - \\
\hline Chur & $\mathrm{CHU}$ & 759465 & 193152 & 556 & $1950-2018$ & - & $1950-2018$ & - \\
\hline Delémont & DEM & 593269 & 244543 & 439 & $1959-2018$ & - & $1950-2018$ & - \\
\hline Einsiedeln & EIN & 699982 & 221068 & 910 & $1950-2018$ & - & $1950-2018$ & - \\
\hline Elm & ELM & 732265 & 198425 & 957 & $1950-2018$ & $1950-2018$ & $1950-2018$ & $1950-2018$ \\
\hline Engelberg & ENG & 674160 & 186069 & 1035 & $1950-2018$ & $1950-2018$ & $1950-2018$ & $1950-2018$ \\
\hline Glarus & GLA & 723755 & 210567 & 516 & $1950-2018$ & - & $1950-2018$ & - \\
\hline Grächen & GRC & 630738 & 116062 & 1605 & $1950-2018$ & $1950-2018$ & $1950-2018$ & $1950-2018$ \\
\hline Grimsel-Hospiz & GRH & 668583 & 158215 & 1980 & $1950-2018$ & $1950-2018$ & $1950-2018$ & $1950-2018$ \\
\hline Col-du-Grand-St-Bernard & GSB & 579192 & 79753 & 2472 & $1950-2018$ & $1950-2018$ & $1950-2018$ & - \\
\hline Genève-Cointrin & GVE & 498904 & 122631 & 410 & $1954-2018$ & $1954-2018$ & $1950-2018$ & $1954-2018$ \\
\hline Hallau & HLL & 677456 & 283472 & 419 & $1959-2018$ & - & $1950-2018$ & - \\
\hline Interlaken & INT & 633023 & 169092 & 577 & $1950-2018$ & - & $1950-2018$ & - \\
\hline Zurich-Kloten & KLO & 682710 & 259338 & 426 & $1950-2018$ & - & $1950-2018$ & - \\
\hline Koppigen & KOP & 612662 & 218664 & 485 & $1961-2018$ & - & $1961-2018$ & - \\
\hline Langnau-i.E. & LAG & 628003 & 198793 & 743 & $1950-2018$ & - & $1950-2018$ & - \\
\hline Luzern & LUZ & 665543 & 209849 & 454 & $1950-2018$ & $1950-2018$ & $1950-2018$ & $1950-2018$ \\
\hline Meiringen & MER & 655844 & 175930 & 588 & $1950-2018$ & $1950-2018$ & $1950-2018$ & $1950-2018$ \\
\hline Mühleberg & MUB & 587792 & 202479 & 479 & $1988-2018$ & - & $1988-2018$ & - \\
\hline Napf & NAP & 638136 & 206078 & 1403 & $1978-2018$ & - & $1978-2018$ & - \\
\hline Neuchâtel & NEU & 563086 & 205559 & 485 & $1950-2018$ & - & $1950-2018$ & - \\
\hline Locarno-Monti & OTL & 704172 & 114342 & 366 & $1950-2018$ & $1950-2018$ & $1950-2018$ & $1950-2018$ \\
\hline Payerne & PAY & 562131 & 184611 & 490 & $1965-2018$ & - & $1965-2018$ & - \\
\hline Bad-Ragaz & RAG & 756910 & 209350 & 496 & $1950-2018$ & $1950-2018$ & $1950-2018$ & - \\
\hline Santis & SAE & 744183 & 234918 & 2502 & $1950-2018$ & - & $1950-2018$ & $1950-2018$ \\
\hline Samedan & SAM & 787249 & 155685 & 1708 & $1979-2018$ & - & $1980-2018$ & $1979-2018$ \\
\hline S.Bernardino & SBE & 734115 & 147294 & 1638 & $1968-2018$ & $1968-2018$ & $1968-2018$ & $1968-2018$ \\
\hline Segl-Maria IA & SIA & 778574 & 144976 & 1804 & $1950-2018$ & - & $1950-2018$ & $1950-2018$ \\
\hline Sion & $\mathrm{SIO}$ & 591633 & 118583 & 482 & $1958-2018$ & $1958-2018$ & $1958-2018$ & $1958-2018$ \\
\hline Zurich-Fluntern & SMA & 747865 & 254588 & 775 & $1866-2017$ & $1866-2017$ & $1890-2017$ & $1866-2017$ \\
\hline St.Gallen & STG & 747865 & 254588 & 775 & $1950-2018$ & $1950-2018$ & $1950-2018$ & $1950-2018$ \\
\hline Aadorf-Tänikon & TAE & 710517 & 259824 & 539 & $1971-2018$ & - & $1970-2018$ & - \\
\hline Vaduz & VAD & 757722 & 221699 & 457 & $1971-2018$ & - & $1971-2018$ & - \\
\hline Wädenswil & WAE & 693847 & 230744 & 485 & $1981-2018$ & - & 1961-2018 & - \\
\hline Wynau & WYN & 626404 & 233848 & 422 & $1978-2018$ & - & $1978-2018$ & - \\
\hline
\end{tabular}




\section{S1.3 STL analysis details}

In this section, some examples of output of the STL analysis are presented. Figures S1 to S4 show the three different components of the STL (seasonal, trend, residuals) for the FOEN water station Reuss-Meillingen and for the MeteoSwiss station of Luzern. As we can see, the seasonal removal works correctly for water temperature, for discharge and for air temperature. For 5 precipitation, the effect is negligible. The STL has been applied here with $n_{s}=37$.

In Figures S5 and S6, the ACF and PACF of the residuals time series are shown (also with $n_{s}=37$ ). If some seasonal signal still exists in the ACF, it is absent in the PACF, meaning that the data at one-year lag in time have no explanatory power on the current data, which is the goal to be achieved. In addition, this plot shows the absence of strong seasonality in the precipitations, especially, as we can expect, in the PACF, justifying the usage of these time series even if the STL has almost no effect.

Finally, in figures S7 and S8 the evolution of ACF and PACF for stream and air temperature residuals time series and for discharge and precipitation residuals time series for varying values of $n_{s}$ are shown.
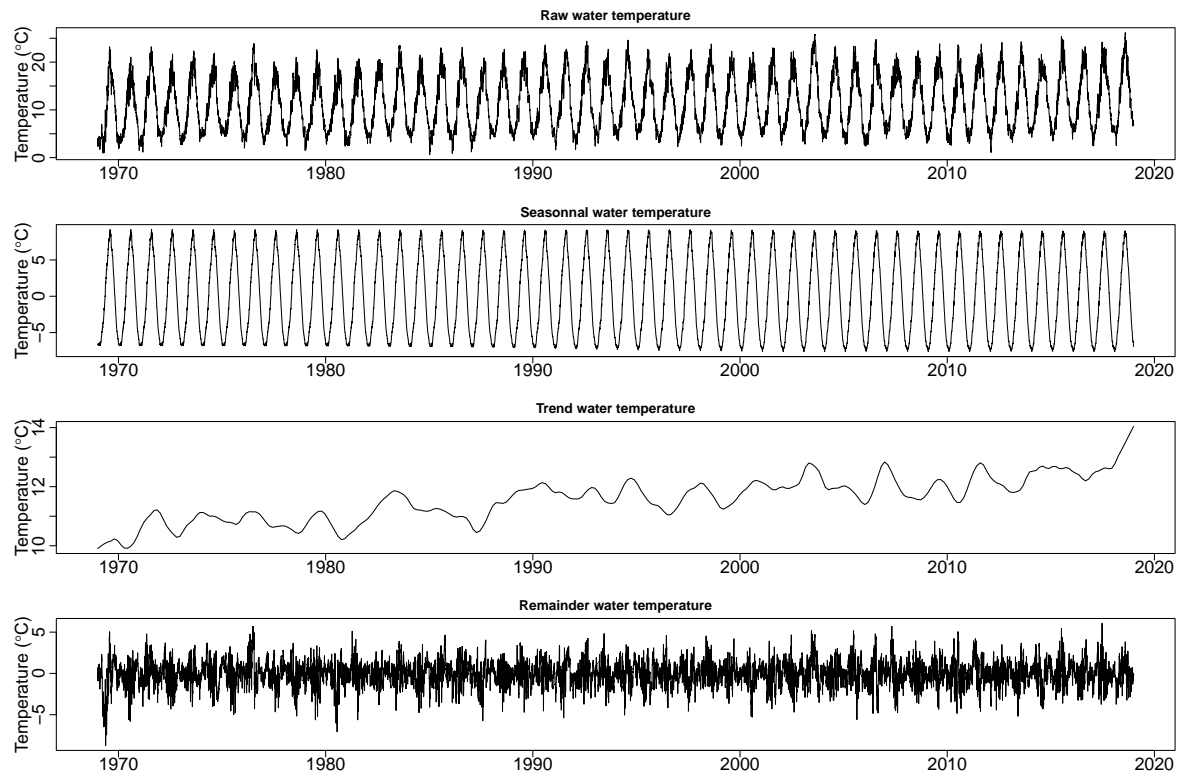

Figure S1. STL decomposition for water temperature for the Reuss river at the FOEN measurement station of Mellingen. Top: Raw data; $2^{\text {nd }}$ row: seasonal part; $3^{\text {rd }}$ row: trend part; bottom: residuals. Series obtained with $n_{s}=37$. 

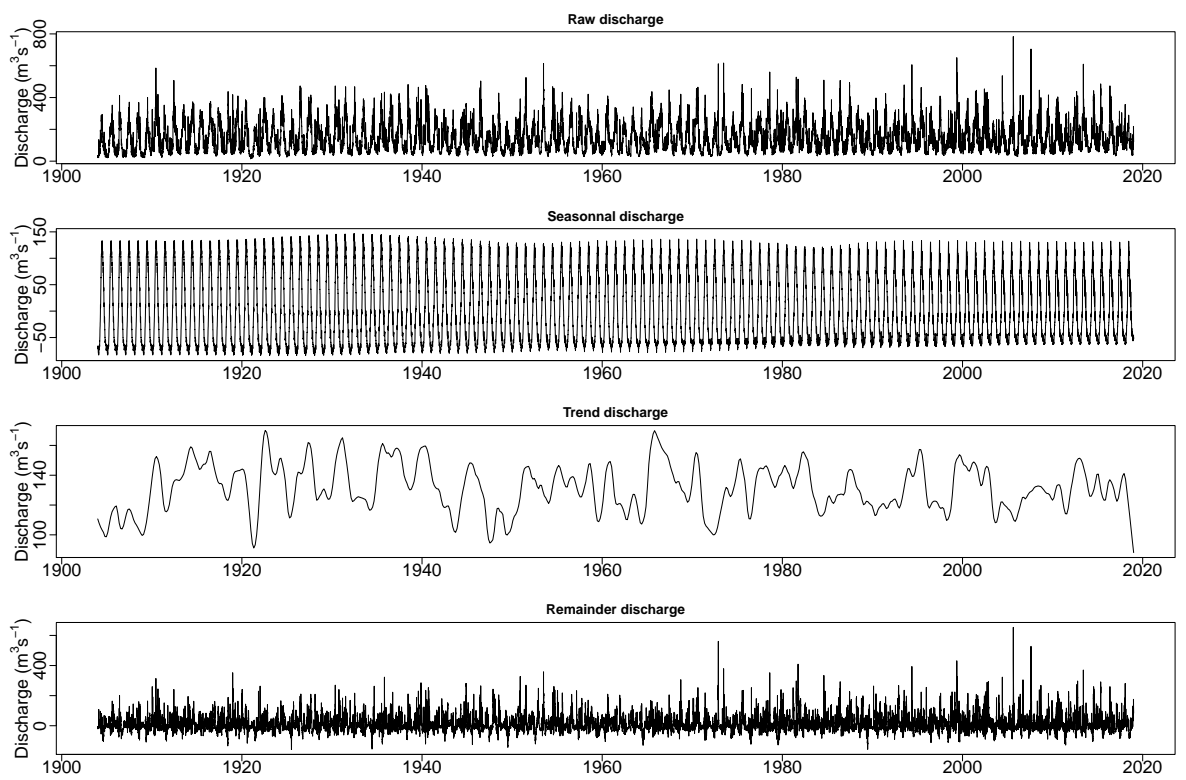

Figure S2. STL decomposition for discharge for the Reuss river at the FOEN measurement station of Mellingen. Top: Raw data; $2^{\text {nd }}$ row: seasonal part; $3^{\text {rd }}$ row: trend part; bottom: residuals. Series obtained with $n_{s}=37$.
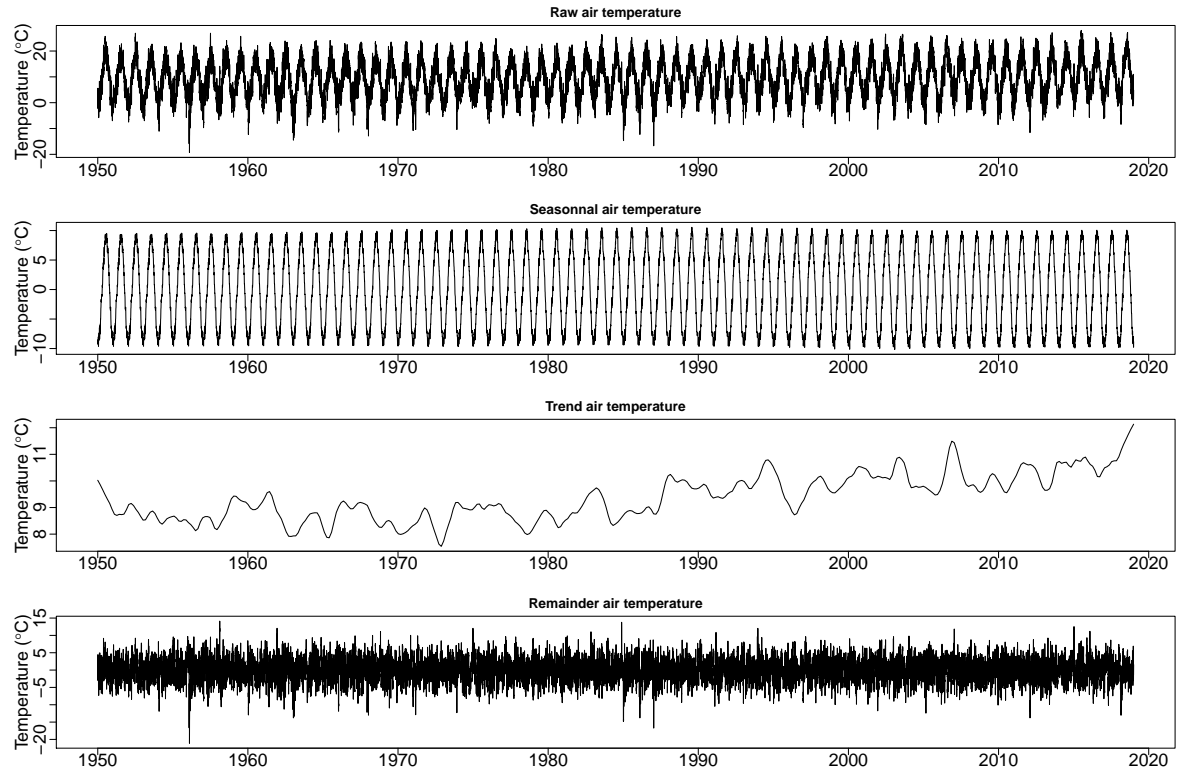

Figure S3. STL decomposition for air temperature for the MeteoSwiss measurement station of Luzern. Top: Raw data; $2^{\text {nd }}$ row: seasonal part; $3^{\text {rd }}$ row: trend part; bottom: residuals. Series obtained with $n_{s}=37$. 

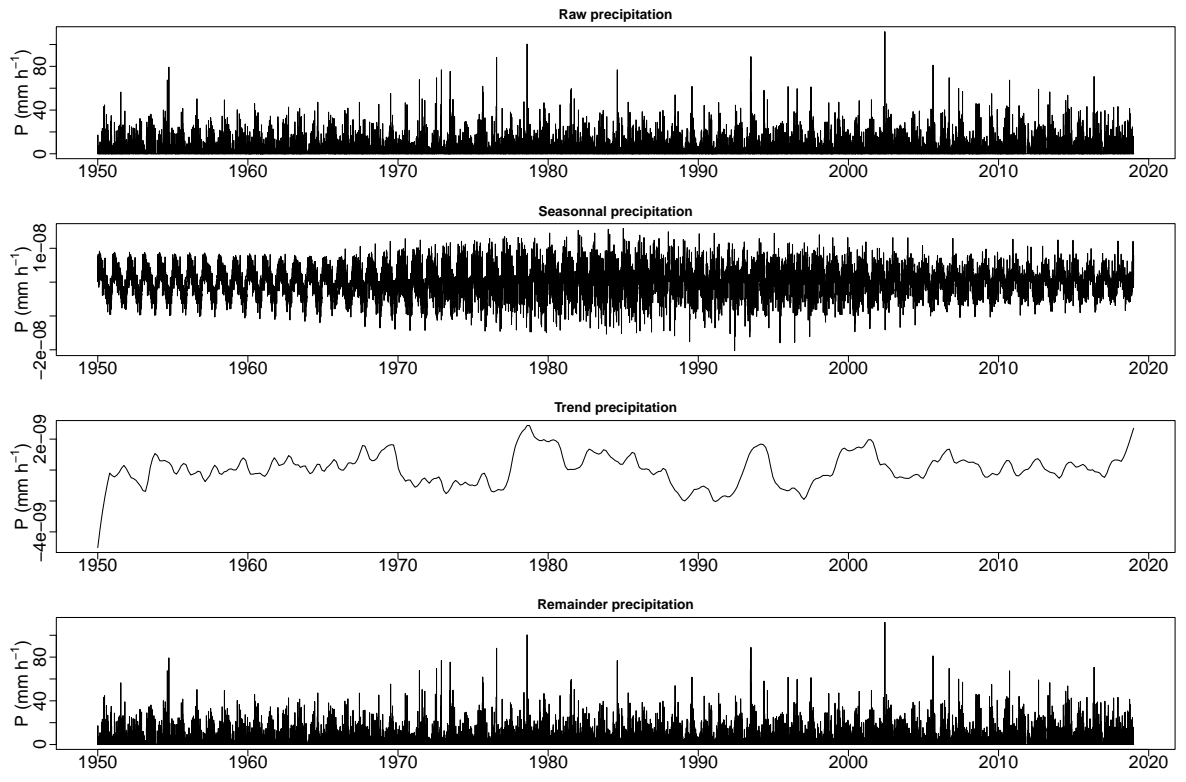

Figure S4. STL decomposition for precipitation for the MeteoSwiss measurement station of Luzern. Top: Raw data; $2^{\text {nd }}$ row: seasonal part; $3^{\text {rd }}$ row: trend part; bottom: residuals. Series obtained with $n_{s}=37$.
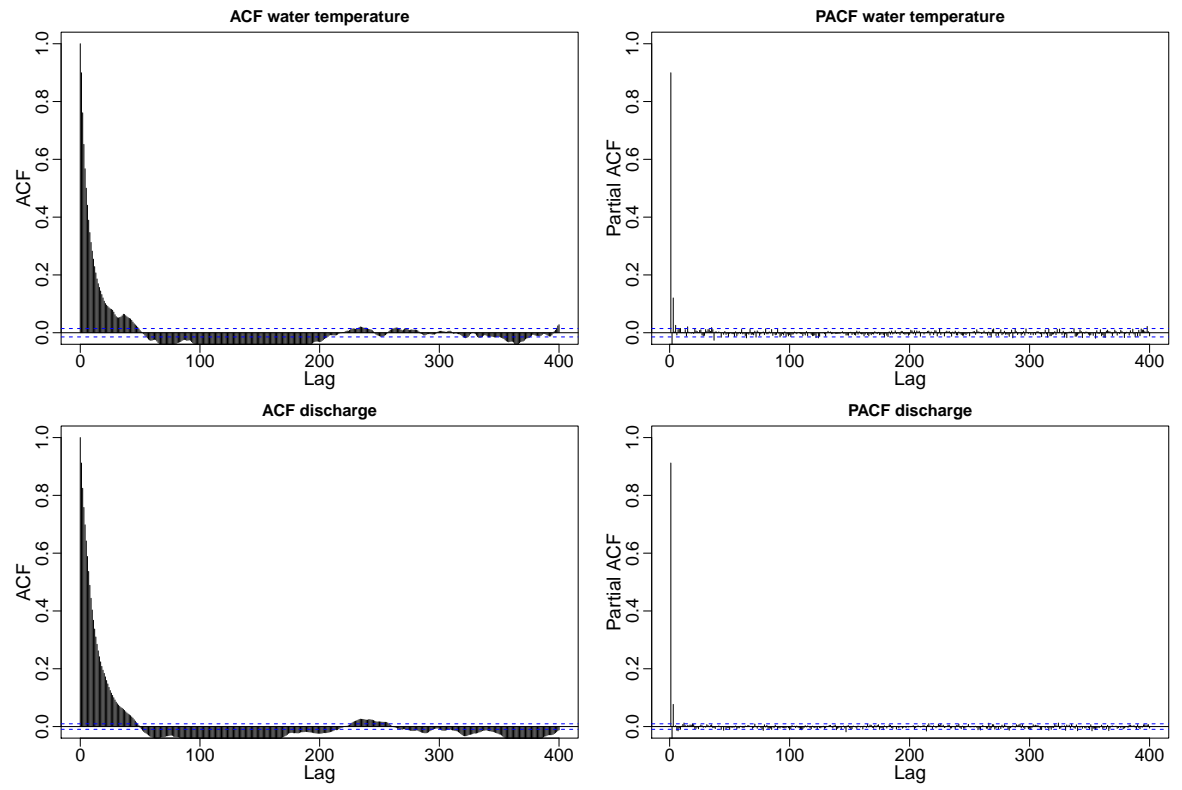

Figure S5. ACF and PACF of the residuals time series of the STL analysis for water temperature (top) and discharge (bottom) for the Reuss river at the FOEN measurement station of Mellingen. 

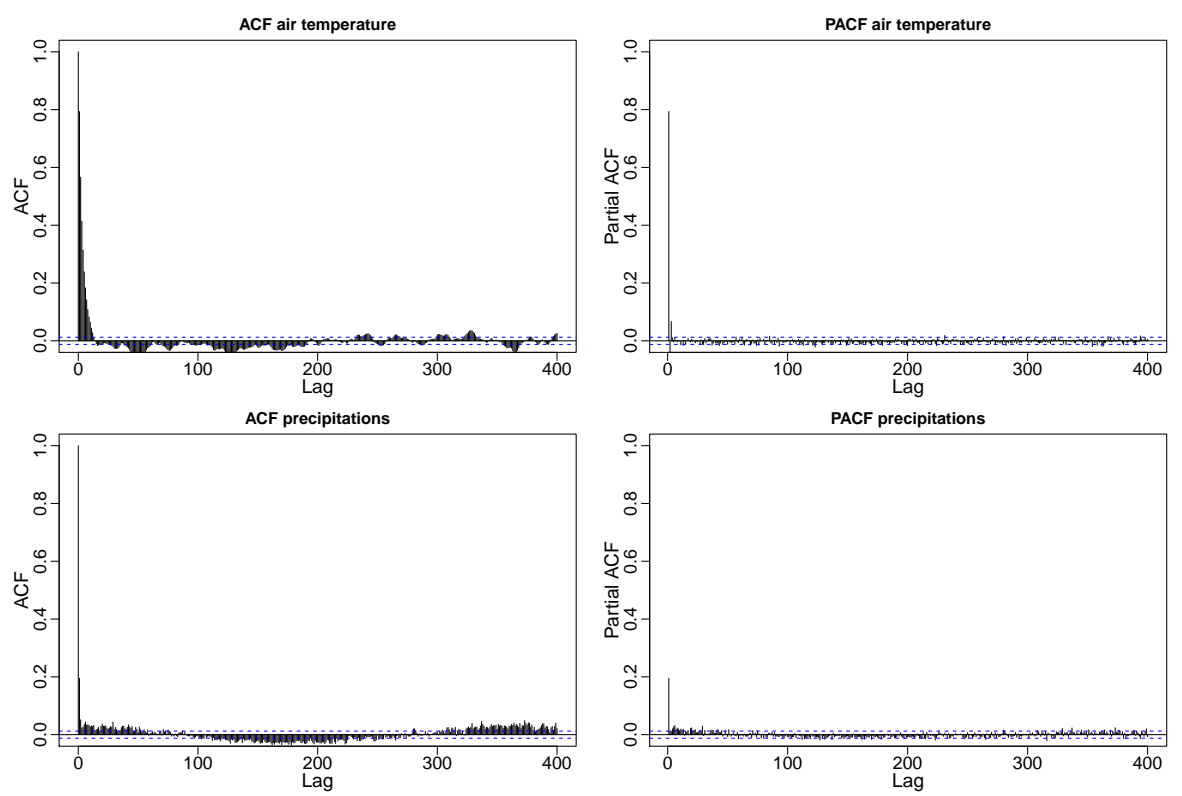

Figure S6. ACF and PACF of the residuals time series of the STL analysis for air temperature (top) and precipitation (bottom) for the MeteoSwiss measurement station of Luzern.

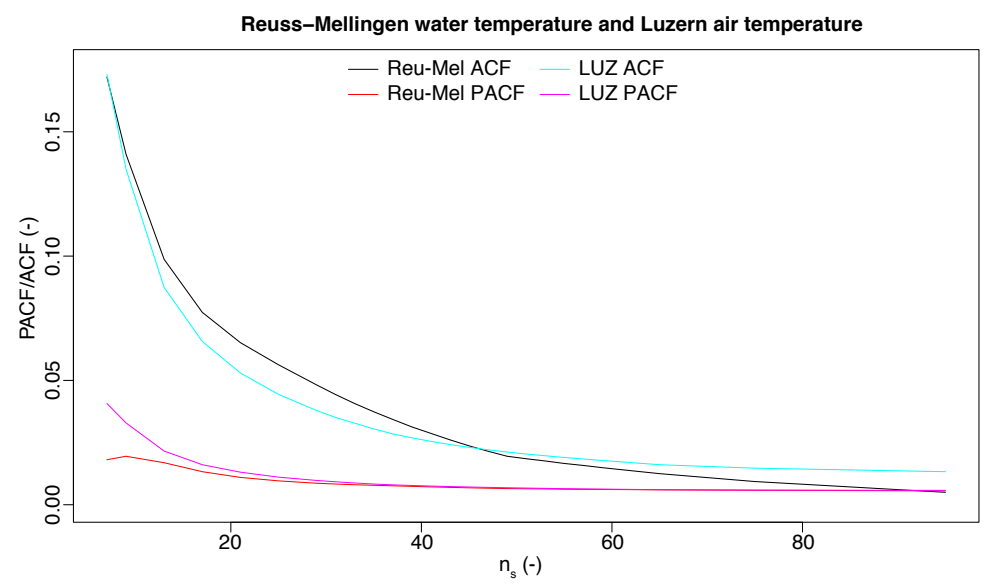

Figure S7. Evolution of the ACF and PACF of the residuals time series of the STL analysis for varying values of $n_{s}$ for the Reuss water temperature at the FOEN measurement station of Mellingen and for air temperature at the MeteoSwiss measurement station of Luzern. 


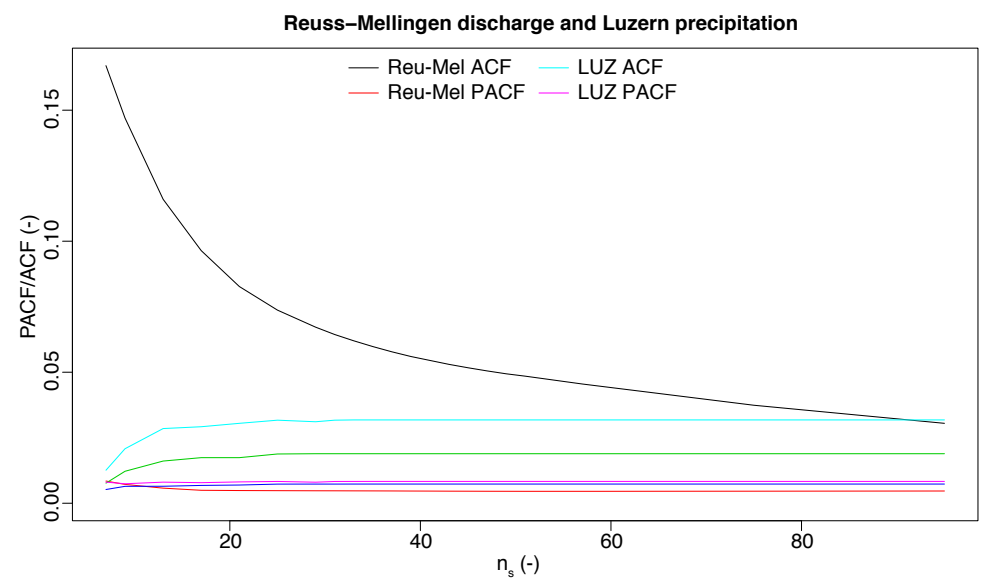

Figure S8. Evolution of the ACF and PACF of the residuals time series of the STL analysis for varying values of $n_{s}$ for the Reuss discharge at the FOEN measurement station of Mellingen and for precipitation at the MeteoSwiss measurement station of Luzern. 


\section{S2 Supplementary material additional results}

\section{S2.1 long-term and trend analysis}

This Section presents additional results for Sections 4.1 and 4.2 of the main article. Figure S9 shows the decadal mean of air temperature anomaly (similar to Figure 2 bottom panel and Figure 4 in the main text).

Figure S10 show the evolution of the climatic indices used in Lehre Seip et al. (2019). These indices are the North Atlantic Oscillation (NAO) (Jones et al., 1997) and the Atlantic Multi-decadal Oscillation (AMO) (Enfield et al., 2001). Data are obtained from the NOAA website. In the Figure, long-term decadal anomalies in precipitation and discharges are shown for comparison.

Tables S3 and S4 show the trends for air temperature and precipitation for the MeteoSwiss station used (see Table S2), for the periods 1999-2018 and 1979-2018. They are similar to Tables A1 and A2 for water temperature and discharge in the Appendix of the main text. Figures S11 and S12 show the same as Figures 5 and 6 in the main text, but for the period 1979-2018.

Finally, Figure S13 shows the distribution of area for the four different alpine regimes and the distribution of the SPJ water temperature trends as function of the catchment area, showing that there is no correlation between the observed water temperature trend and the catchment area (only SPJ catchments are plotted to separate the effect of regime and the effect of area).

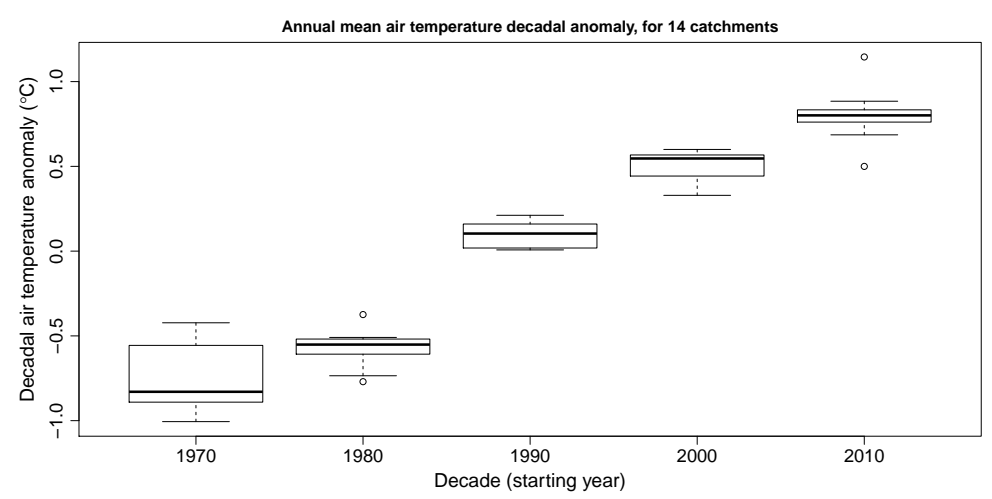

Figure S9. Air temperature anomalies per decade with respect to the $1970-2018$ mean, for the 14 catchments with data available since 1970 (same catchments as for water temperature in Figure 2 bottom panel in main text). 

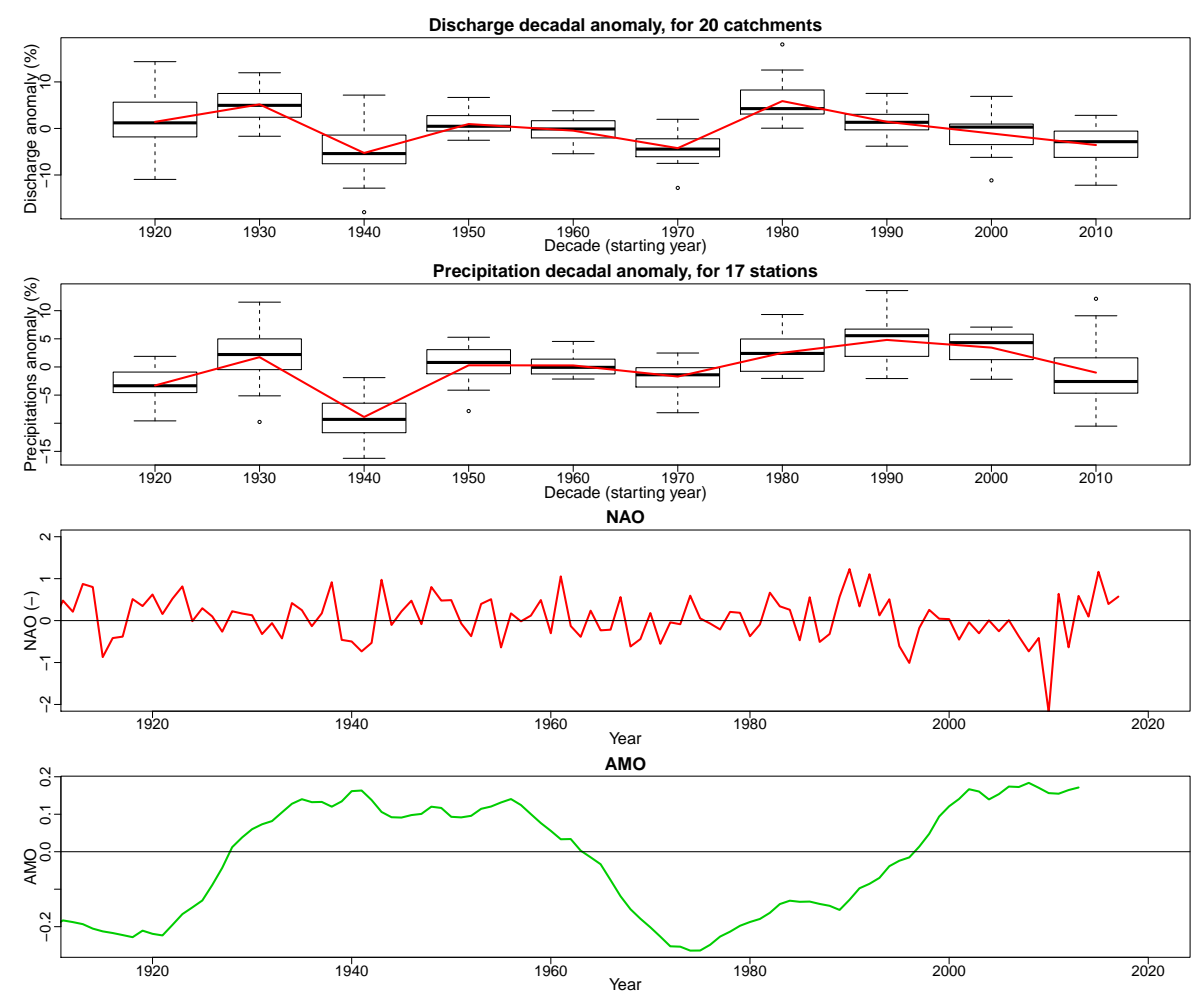

Figure S10. Relative discharge and precipitation decadal means of anomalies with respect to the 1920-2018 average for 20 catchments and 22 MeteoSwiss homogeneous stations with data available since 1920 (upper two plots). Yearly mean of the NAO and AMO (lower two plots).
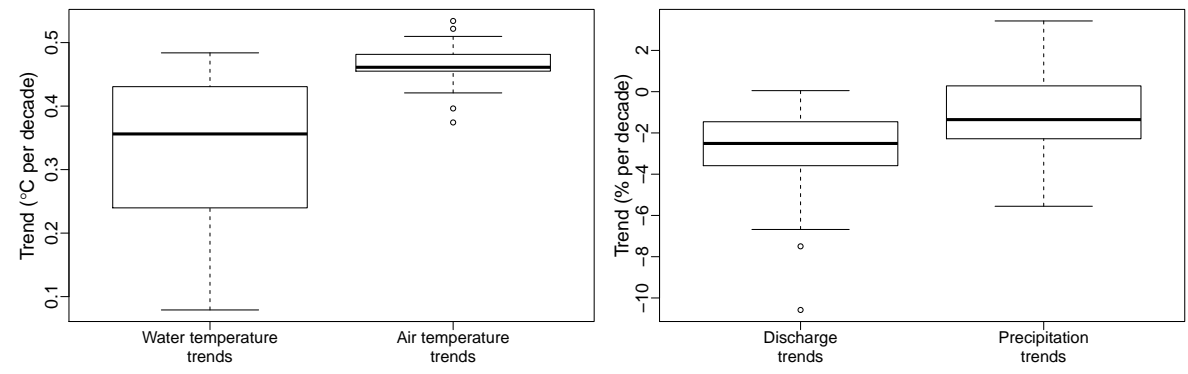

Figure S11. Distributions of trends of water and air temperature (left), and normalized discharge and normalized precipitation (right), for the periods 1979-2018 for the 27 catchments where data are available for temperature and discharge (see Table 1 in main text). 
Table S3. Air temperature (left part) and precipitation (right part) annual and seasonal trends for all the MeteoSwiss stations presented in Table S2 over the period 1999-2018. The numbers in brackets indicate the standard error of the computed trends based on linear regression.

\begin{tabular}{|c|c|c|c|c|c|c|c|c|c|c|}
\hline \multirow[b]{2}{*}{ River Name } & \multicolumn{5}{|c|}{ Water temperature trend $\left({ }^{\circ}\right.$ per decade) } & \multicolumn{5}{|c|}{ Discharge trend (\% per decade) } \\
\hline & Annual & Winter & Spring & Summer & Fall & Annual & Winter & Spring & Summer & Fall \\
\hline $\mathrm{ABO}$ & $0.44(0.08)$ & $0.32(0.63)$ & $0.15(0.39)$ & $0.56(0.40)$ & $0.61(0.43)$ & $-6.9(4.1)$ & $-3.1(14.5)$ & $-7.4(11.5)$ & $-11.7(8.1)$ & $-10.8(11.4)$ \\
\hline ALT & $0.33(0.07)$ & $0.20(0.45)$ & $0.13(0.35)$ & $0.49(0.39)$ & $0.43(0.37)$ & $-8.9(4.5)$ & $-6.4(13.4)$ & $-13.3(11.5)$ & $-6.2(8.1)$ & $-16.6(14.3)$ \\
\hline BAS & $0.35(0.07)$ & $0.09(0.54)$ & $0.14(0.40)$ & $0.70(0.43)$ & $0.35(0.35)$ & $-8.5(4.5)$ & $18.2(10.6)$ & $-8.5(15.5)$ & $-10.5(11.9)$ & $-28.6(14.3)$ \\
\hline BEH & $0.13(0.08)$ & $0.26(0.63)$ & $-0.23(0.46)$ & $0.01(0.52)$ & $0.40(0.38)$ & $-37.7(5.3)$ & $-39.9(41.3)$ & $-55.1(23.4)$ & $-9.7(10.7)$ & $-43.1(20.2)$ \\
\hline BER & $0.42(0.07)$ & $0.22(0.50)$ & $0.17(0.37)$ & $0.75(0.38)$ & $0.44(0.34)$ & $-12.5(4.5)$ & $13.4(10.9)$ & $-16.3(14.8)$ & $-17.1(10.4)$ & $-25.5(12.6)$ \\
\hline $\mathrm{CDF}$ & $0.51(0.08)$ & $0.35(0.57)$ & $0.41(0.39)$ & $0.69(0.38)$ & $0.50(0.43)$ & $-9.2(4)$ & $5.1(11.8)$ & $-10.1(15.1)$ & $-8.8(10.8)$ & $-28.8(13.5)$ \\
\hline CHA & $0.53(0.09)$ & $0.25(0.62)$ & $0.43(0.46)$ & $0.60(0.46)$ & $0.66(0.49)$ & $-3.1(4.3)$ & $-21.3(14.3)$ & $-5.7(17.1)$ & $14.4(14.5)$ & $-6.0(14.5)$ \\
\hline $\mathrm{CHU}$ & $0.50(0.07)$ & $0.43(0.57)$ & $0.20(0.37)$ & $0.73(0.42)$ & $0.58(0.42)$ & $-12.0(5.2)$ & $-9.0(17.2)$ & $-11.4(15.2)$ & $-15.3(10.3)$ & $-20.3(13.9)$ \\
\hline DEM & $0.01(0.07)$ & $-0.07(0.53)$ & $-0.23(0.39)$ & $0.27(0.40)$ & $0.00(0.35)$ & $-16.4(4.5)$ & $0.0(10.1)$ & $-18.4(14.9)$ & $-12.4(10.1)$ & $-36.2(16)$ \\
\hline EIN & $0.49(0.08)$ & $0.32(0.57)$ & $0.28(0.40)$ & $0.71(0.39)$ & $0.52(0.40)$ & $-16.0(4)$ & $-21.6(12.3)$ & $-21.2(9.9)$ & $-11.6(9.1)$ & $-18.1(11.7)$ \\
\hline ELM & $0.52(0.08)$ & $0.46(0.57)$ & $0.36(0.39)$ & $0.61(0.37)$ & $0.59(0.43)$ & $-10.8(4)$ & $-13.5(13.1)$ & $-11.8(10.1)$ & $-10.3(8.3)$ & $-16.3(12.8)$ \\
\hline ENG & $0.51(0.08)$ & $0.41(0.58)$ & $0.28(0.39)$ & $0.63(0.38)$ & $0.61(0.42)$ & $-5.1(3.9)$ & $-6.4(13)$ & $-7.4(9.9)$ & $-3.6(7.4)$ & $-9.9(12)$ \\
\hline GLA & $0.43(0.07)$ & $0.30(0.55)$ & $0.29(0.39)$ & $0.58(0.39)$ & $0.42(0.38)$ & $-9.5(4.1)$ & $-7.4(13.9)$ & $-15.8(11.2)$ & $-8.1(9.1)$ & $-15.0(11.8)$ \\
\hline GRC & $0.50(0.07)$ & $0.33(0.59)$ & $0.27(0.39)$ & $0.67(0.39)$ & $0.63(0.40)$ & $-14.1(6.2)$ & $8.0(16.9)$ & $-10.6(13.6)$ & $-17.7(12.7)$ & $-37.2(24.5)$ \\
\hline GRH & $0.43(0.08)$ & $0.50(0.61)$ & $0.25(0.40)$ & $0.41(0.37)$ & $0.52(0.45)$ & $-6.0(4.2)$ & $-12.1(18.3)$ & $5.6(9.4)$ & $-7.7(9)$ & $-13.1(12.5)$ \\
\hline GSB & $0.45(0.08)$ & $0.22(0.58)$ & $0.21(0.37)$ & $0.59(0.40)$ & $0.65(0.35)$ & $-10.5(4.3)$ & $2.1(13.7)$ & $-8.8(8.1)$ & $-22.7(10.5)$ & $-18.2(14.3)$ \\
\hline GVE & $0.33(0.06)$ & $0.17(0.45)$ & $0.14(0.37)$ & $0.54(0.45)$ & $0.41(0.35)$ & $-16.0(4.8)$ & $11.1(11.4)$ & $-16.6(16)$ & $-21.1(9.9)$ & $-35.3(12.9)$ \\
\hline HLL & $0.13(0.07)$ & $0.07(0.48)$ & $-0.19(0.39)$ & $0.37(0.41)$ & $0.19(0.32)$ & $-25.4(4.5)$ & $-20.0(10.4)$ & $-30.4(15.4)$ & $-17.3(10.8)$ & $-35.6(12.9)$ \\
\hline INT & $0.51(0.06)$ & $0.39(0.43)$ & $0.35(0.34)$ & $0.71(0.36)$ & $0.52(0.29)$ & $-3.6(4.3)$ & $1.9(13.8)$ & $-3.8(10.9)$ & $-5.5(11.1)$ & $-11.9(12.6)$ \\
\hline KLO & $0.41(0.07)$ & $0.17(0.49)$ & $0.14(0.36)$ & $0.73(0.41)$ & $0.49(0.33)$ & $-14.7(4.4)$ & $-2.7(9.6)$ & $-18.5(13.8)$ & $-15.7(10.2)$ & $-24.6(12)$ \\
\hline KOP & $0.13(0.07)$ & $0.05(0.51)$ & $-0.16(0.35)$ & $0.43(0.38)$ & $0.12(0.33)$ & $-13.2(4.4)$ & $1.3(9.7)$ & $-9.7(14.8)$ & $-15.8(11.1)$ & $-28.1(14)$ \\
\hline LAG & $0.25(0.07)$ & $0.00(0.51)$ & $0.07(0.37)$ & $0.65(0.35)$ & $0.18(0.36)$ & $-11.2(4.2)$ & $1.1(9.8)$ & $-9.1(11.4)$ & $-15.3(9.7)$ & $-18.4(10.7)$ \\
\hline LUZ & $0.39(0.07)$ & $0.23(0.46)$ & $0.17(0.37)$ & $0.63(0.41)$ & $0.45(0.32)$ & $-2.0(4.3)$ & $19.9(10.4)$ & $-0.4(12.2)$ & $-9.9(9.2)$ & $-7.4(10.4)$ \\
\hline MER & $0.46(0.07)$ & $0.38(0.47)$ & $0.34(0.35)$ & $0.57(0.35)$ & $0.45(0.35)$ & $-10.5(4.2)$ & $-11.7(15.4)$ & $-15.1(11.2)$ & $-7.5(8)$ & $-14.4(14.7)$ \\
\hline MUB & $0.40(0.06)$ & $0.10(0.48)$ & $0.19(0.37)$ & $0.77(0.39)$ & $0.49(0.33)$ & $-7.6(4.5)$ & $13.3(10)$ & $-10.8(14.9)$ & $-10.9(13.4)$ & $-18.3(13.6)$ \\
\hline NAP & $0.53(0.09)$ & $0.35(0.65)$ & $0.34(0.46)$ & $0.64(0.47)$ & $0.64(0.51)$ & $-8.3(4)$ & $2.8(14.6)$ & $-11.5(10.7)$ & $-11.5(9.4)$ & $-12.6(13.2)$ \\
\hline $\mathrm{NEU}$ & $0.38(0.07)$ & $0.13(0.49)$ & $0.18(0.42)$ & $0.63(0.44)$ & $0.50(0.34)$ & $-9.9(4.6)$ & $8.0(10.6)$ & $-3.7(17.9)$ & $-15.6(13.3)$ & $-30.1(13.5)$ \\
\hline OTL & $0.47(0.06)$ & $0.40(0.36)$ & $0.24(0.40)$ & $0.61(0.43)$ & $0.61(0.25)$ & $-9.1(5.8)$ & $22.2(27.4)$ & $-1.4(15.4)$ & $-17.9(13.3)$ & $-20.1(18)$ \\
\hline PAY & $0.31(0.07)$ & $0.13(0.49)$ & $0.05(0.36)$ & $0.54(0.42)$ & $0.43(0.33)$ & $-10.4(4.7)$ & $16.7(12.3)$ & $-17.3(15.2)$ & $-9.5(11.9)$ & $-26.7(14.2)$ \\
\hline RAG & $0.30(0.08)$ & $0.46(0.60)$ & $0.04(0.40)$ & $0.37(0.39)$ & $0.23(0.42)$ & $-0.2(4.7)$ & $-6.1(15.4)$ & $5.1(12.7)$ & $-6.0(9.9)$ & $-3.9(14.6)$ \\
\hline SAE & $0.39(0.09)$ & $0.24(0.60)$ & $0.24(0.40)$ & $0.63(0.39)$ & $0.41(0.51)$ & $-7.8(3.9)$ & $-1.1(14.2)$ & $-17.1(14.6)$ & $-7.4(8.1)$ & $-14.2(13)$ \\
\hline SAM & $0.38(0.07)$ & $0.84(0.61)$ & $-0.01(0.32)$ & $0.35(0.31)$ & $0.36(0.37)$ & $-8.6(5.7)$ & $24.5(22.2)$ & $0.0(12)$ & $-7.3(10.5)$ & $-34.5(17.5)$ \\
\hline SBE & $0.48(0.06)$ & $0.35(0.48)$ & $0.32(0.38)$ & $0.52(0.37)$ & $0.62(0.32)$ & $0.0(5)$ & $27.6(25.9)$ & $7.0(12.8)$ & $-2.9(10.6)$ & $-16.4(17.8)$ \\
\hline SIA & $0.42(0.06)$ & $0.51(0.51)$ & $0.19(0.33)$ & $0.56(0.32)$ & $0.40(0.31)$ & $-12.5(5.4)$ & $6.3(21.5)$ & $-7.1(12.6)$ & $-11.9(9.6)$ & $-28.5(18.8)$ \\
\hline SIO & $0.67(0.06)$ & $0.42(0.50)$ & $0.37(0.33)$ & $1.03(0.37)$ & $0.78(0.32)$ & $-4.9(5.6)$ & $23.2(15)$ & $-8.3(17.9)$ & $-19.1(11.3)$ & $-21.7(18.2)$ \\
\hline SMA & $0.38(0.07)$ & $0.10(0.53)$ & $0.15(0.42)$ & $0.70(0.43)$ & $0.46(0.35)$ & $-11.7(4.4)$ & $-1.1(10.1)$ & $-21.1(12.9)$ & $-5.3(9.9)$ & $-23.2(12)$ \\
\hline STG & $0.33(0.08)$ & $0.17(0.64)$ & $0.14(0.43)$ & $0.50(0.41)$ & $0.40(0.41)$ & $0.3(4.4)$ & $22.5(12.2)$ & $-8.8(11.4)$ & $-0.4(8.2)$ & $-7.9(11)$ \\
\hline TAE & $0.42(0.07)$ & $0.24(0.51)$ & $0.11(0.34)$ & $0.71(0.38)$ & $0.49(0.35)$ & $-9.9(4.2)$ & $-1.3(9.7)$ & $-17.6(12.5)$ & $-5.0(8.9)$ & $-18.1(14.2)$ \\
\hline VAD & $0.52(0.08)$ & $0.35(0.60)$ & $0.24(0.39)$ & $0.79(0.39)$ & $0.55(0.42)$ & $-8.0(4.9)$ & $-9.6(13.8)$ & $-9.0(11.8)$ & $-9.6(6.6)$ & $-10.2(13.4)$ \\
\hline WAE & $0.46(0.07)$ & $0.32(0.50)$ & $0.20(0.39)$ & $0.68(0.42)$ & $0.54(0.34)$ & $-9.5(4.2)$ & $-0.7(11.1)$ & $-15.8(11.4)$ & $-7.0(9.1)$ & $-20.8(11.8)$ \\
\hline WYN & $0.43(0.06)$ & $0.28(0.49)$ & $0.20(0.34)$ & $0.69(0.40)$ & $0.47(0.34)$ & $-8.1(4.4)$ & $4.5(11.3)$ & $-5.4(15.3)$ & $-8.9(9.4)$ & $-23.8(12.4)$ \\
\hline
\end{tabular}


Table S4. Air temperature (left part) and precipitation (right part) annual and seasonal trends for all the MeteoSwiss stations presented in Table S2 over the period 1979-2018. The numbers in brackets indicate the standard error of the computed trends based on linear regression.

\begin{tabular}{|c|c|c|c|c|c|c|c|c|c|c|}
\hline \multirow[b]{2}{*}{ River Name } & \multicolumn{5}{|c|}{ Water temperature trend $\left({ }^{\circ}\right.$ per decade $)$} & \multicolumn{5}{|c|}{ Discharge trend (\% per decade) } \\
\hline & Annual & Winter & Spring & Summer & Fall & Annual & Winter & Spring & Summer & Fall \\
\hline $\mathrm{ABO}$ & $0.36(0.03)$ & $0.14(0.21)$ & $0.60(0.14)$ & $0.46(0.12)$ & $0.22(0.14)$ & $-3.9(1.4)$ & $-9.5(4.4)$ & $-1.8(3.4)$ & $-0.9(2.6)$ & $-7.9(4.2)$ \\
\hline ALT & $0.48(0.02)$ & $0.28(0.17)$ & $0.64(0.12)$ & $0.61(0.12)$ & $0.35(0.12)$ & $0.3(1.6)$ & $-4.5(4.3)$ & $-0.2(3.7)$ & $3.1(2.6)$ & $-1.9(4.8)$ \\
\hline BAS & $0.49(0.03)$ & $0.34(0.20)$ & $0.59(0.13)$ & $0.66(0.13)$ & $0.35(0.12)$ & $1.1(1.6)$ & $0.1(4)$ & $1.8(5)$ & $1.7(3.8)$ & $-0.8(4.6)$ \\
\hline BEH & $0.26(0.03)$ & $0.28(0.21)$ & $0.48(0.17)$ & $0.18(0.15)$ & $0.11(0.14)$ & $-8.6(1.9)$ & $-4.1(11.1)$ & $-20.8(7.5)$ & $-3.0(4.7)$ & $-5.5(6.5)$ \\
\hline BER & $0.48(0.02)$ & $0.28(0.18)$ & $0.61(0.13)$ & $0.65(0.11)$ & $0.34(0.11)$ & $-3.9(1.6)$ & $-2.4(4.3)$ & $-3.5(4.5)$ & $-2.6(3.9)$ & $-8.8(4.1)$ \\
\hline $\mathrm{CDF}$ & $0.49(0.03)$ & $0.23(0.20)$ & $0.73(0.13)$ & $0.63(0.12)$ & $0.34(0.14)$ & $-4.6(1.4)$ & $-5.4(4.2)$ & $-5.4(4.7)$ & $-1.2(3.7)$ & $-8.7(4.5)$ \\
\hline CHA & $0.36(0.03)$ & $0.06(0.24)$ & $0.66(0.16)$ & $0.50(0.15)$ & $0.22(0.18)$ & $-0.4(1.6)$ & $-11.5(5.8)$ & $1.9(5.9)$ & $11.6(5.2)$ & $-4.9(5.5)$ \\
\hline $\mathrm{CHU}$ & $0.59(0.03)$ & $0.40(0.20)$ & $0.72(0.13)$ & $0.76(0.13)$ & $0.45(0.13)$ & $-0.9(1.9)$ & $-4.1(5.7)$ & $-2.9(4.9)$ & $0.9(3.9)$ & $-1.8(5.2)$ \\
\hline DEM & $0.43(0.03)$ & $0.32(0.20)$ & $0.54(0.14)$ & $0.54(0.12)$ & $0.26(0.12)$ & $-4.4(1.5)$ & $-6.2(3.6)$ & $-7.4(4.7)$ & $-0.2(3.4)$ & $-6.1(5)$ \\
\hline EIN & $0.43(0.03)$ & $0.28(0.20)$ & $0.60(0.14)$ & $0.54(0.12)$ & $0.26(0.13)$ & $-4.9(1.4)$ & $-8.6(3.9)$ & $-3.4(3.4)$ & $-3.1(2.9)$ & $-7.7(4.2)$ \\
\hline ELM & $0.48(0.03)$ & $0.30(0.20)$ & $0.70(0.13)$ & $0.56(0.11)$ & $0.31(0.14)$ & $-2.3(1.4)$ & $-4.9(4.3)$ & $-2.9(3.4)$ & $-0.4(2.9)$ & $-3.6(4.6)$ \\
\hline ENG & $0.43(0.03)$ & $0.21(0.20)$ & $0.61(0.14)$ & $0.56(0.12)$ & $0.30(0.14)$ & $0.1(1.4)$ & $-4.4(3.9)$ & $2.2(3.1)$ & $2.4(2.3)$ & $-3.4(4.2)$ \\
\hline GLA & $0.44(0.03)$ & $0.27(0.18)$ & $0.61(0.13)$ & $0.57(0.12)$ & $0.27(0.12)$ & $-0.6(1.4)$ & $-4.4(4.6)$ & $-1.2(3.7)$ & $1.0(2.9)$ & $-1.4(4.2)$ \\
\hline GRC & $0.45(0.03)$ & $0.12(0.20)$ & $0.75(0.14)$ & $0.60(0.12)$ & $0.30(0.13)$ & $-7.1(2.2)$ & $-10.8(5.5)$ & $-10.1(5.3)$ & $1.9(4.2)$ & $-12.1(6.9)$ \\
\hline GRH & $0.43(0.03)$ & $0.22(0.20)$ & $0.68(0.14)$ & $0.58(0.12)$ & $0.20(0.15)$ & $-2.8(1.5)$ & $-6.6(5.4)$ & $-1.6(3.4)$ & $-1.3(3)$ & $-0.7(4.7)$ \\
\hline GSB & $0.41(0.03)$ & $0.17(0.20)$ & $0.66(0.14)$ & $0.57(0.12)$ & $0.22(0.14)$ & $-2.5(1.5)$ & $-0.5(4.5)$ & $-4.5(3.8)$ & $-0.9(3.7)$ & $-4.9(4.7)$ \\
\hline GVE & $0.46(0.02)$ & $0.21(0.16)$ & $0.62(0.12)$ & $0.60(0.13)$ & $0.34(0.11)$ & $-5.6(1.7)$ & $-9.5(4.6)$ & $-4.7(5.2)$ & $-1.3(4.6)$ & $-8.5(5.1)$ \\
\hline HLL & $0.41(0.02)$ & $0.38(0.18)$ & $0.48(0.14)$ & $0.44(0.13)$ & $0.30(0.11)$ & $-6.9(1.6)$ & $-10.3(4.3)$ & $-5.8(5.4)$ & $-0.2(3.8)$ & $-13.1(4.6)$ \\
\hline INT & $0.52(0.02)$ & $0.26(0.15)$ & $0.70(0.11)$ & $0.69(0.11)$ & $0.40(0.10)$ & $0.1(1.5)$ & $-3.7(4.5)$ & $1.0(3.3)$ & $3.1(3.4)$ & $-3.8(4.4)$ \\
\hline KLO & $0.45(0.02)$ & $0.34(0.18)$ & $0.56(0.13)$ & $0.57(0.12)$ & $0.28(0.11)$ & $1.8(1.6)$ & $-0.9(4)$ & $4.4(5.1)$ & $3.5(3.6)$ & $-3.3(4.6)$ \\
\hline KOP & $0.34(0.02)$ & $0.28(0.19)$ & $0.44(0.13)$ & $0.38(0.12)$ & $0.21(0.11)$ & $-5.0(1.5)$ & $-10.1(3.7)$ & $-4.2(4.4)$ & $-1.0(3.8)$ & $-8.2(4.6)$ \\
\hline LAG & $0.31(0.02)$ & $0.23(0.18)$ & $0.45(0.13)$ & $0.32(0.11)$ & $0.19(0.11)$ & $-6.8(1.4)$ & $-12.2(3.8)$ & $-4.9(3.9)$ & $-3.5(3.2)$ & $-10.2(3.6)$ \\
\hline LUZ & $0.48(0.02)$ & $0.28(0.17)$ & $0.63(0.13)$ & $0.62(0.12)$ & $0.33(0.11)$ & $2.4(1.5)$ & $-1.1(4)$ & $4.8(3.7)$ & $4.4(3.2)$ & $-2.4(3.7)$ \\
\hline MER & $0.50(0.02)$ & $0.36(0.17)$ & $0.67(0.12)$ & $0.57(0.11)$ & $0.33(0.11)$ & $-1.2(1.5)$ & $-9.1(4.3)$ & $-1.7(3.5)$ & $3.5(2.5)$ & $-2.3(4.8)$ \\
\hline MUB & - & - & - & - & - & - & - & - & - & - \\
\hline NAP & $0.44(0.03)$ & $0.12(0.22)$ & $0.72(0.15)$ & $0.63(0.15)$ & $0.24(0.17)$ & $4.5(1.4)$ & $4.6(5.5)$ & $7.5(3.8)$ & $4.7(3.2)$ & $-0.3(4.3)$ \\
\hline NEU & $0.41(0.02)$ & $0.24(0.17)$ & $0.57(0.14)$ & $0.50(0.14)$ & $0.29(0.11)$ & $-2.3(1.6)$ & $-3.8(3.9)$ & $-0.7(5.4)$ & $2.1(4.8)$ & $-9.7(4.4)$ \\
\hline OTL & $0.52(0.02)$ & $0.30(0.12)$ & $0.72(0.13)$ & $0.61(0.12)$ & $0.43(0.08)$ & $-1.5(2.1)$ & $6.4(7.9)$ & $-6.8(5.7)$ & $-2.8(5.1)$ & $1.0(6.6)$ \\
\hline PAY & $0.44(0.02)$ & $0.26(0.17)$ & $0.55(0.13)$ & $0.59(0.12)$ & $0.30(0.11)$ & $-3.9(1.7)$ & $-7.0(5.2)$ & $-3.1(4.9)$ & $-2.3(4.2)$ & $-6.1(4.7)$ \\
\hline RAG & $0.45(0.03)$ & $0.35(0.21)$ & $0.58(0.14)$ & $0.53(0.12)$ & $0.29(0.13)$ & $-1.4(1.7)$ & $-4.7(5)$ & $0.1(4.4)$ & $-2.6(3.1)$ & $-0.5(4.9)$ \\
\hline SAE & $0.35(0.03)$ & $0.08(0.22)$ & $0.62(0.14)$ & $0.54(0.13)$ & $0.13(0.17)$ & $-1.0(1.4)$ & $1.4(4.6)$ & $-3.1(4.6)$ & $0.8(2.4)$ & $-5.1(5.2)$ \\
\hline SAM & $0.52(0.03)$ & $0.59(0.20)$ & $0.60(0.14)$ & $0.51(0.10)$ & $0.39(0.12)$ & $-2.6(2.1)$ & $-0.8(6.5)$ & $-6.9(6.6)$ & $-1.3(3.8)$ & $-3.7(6.3)$ \\
\hline SBE & $0.39(0.02)$ & $0.20(0.17)$ & $0.66(0.13)$ & $0.48(0.11)$ & $0.22(0.11)$ & $-0.5(1.8)$ & $2.4(7.5)$ & $-6.9(5.5)$ & $2.3(4.3)$ & $0.2(6.1)$ \\
\hline SIA & $0.45(0.02)$ & $0.40(0.16)$ & $0.64(0.13)$ & $0.42(0.10)$ & $0.33(0.11)$ & $-2.9(1.9)$ & $-2.4(6.3)$ & $-10.2(5.8)$ & $-1.2(3.9)$ & $-0.5(6.4)$ \\
\hline SIO & $0.63(0.02)$ & $0.33(0.16)$ & $0.74(0.12)$ & $0.80(0.11)$ & $0.61(0.10)$ & $-4.2(2)$ & $-4.9(5.8)$ & $-1.8(5.3)$ & $2.8(3.9)$ & $-16.7(5.3)$ \\
\hline SMA & $0.46(0.03)$ & $0.29(0.19)$ & $0.61(0.14)$ & $0.60(0.13)$ & $0.31(0.12)$ & $-0.9(1.5)$ & $-4.0(3.7)$ & $1.8(4.7)$ & $1.6(3.8)$ & $-6.5(4.6)$ \\
\hline STG & $0.47(0.03)$ & $0.29(0.22)$ & $0.66(0.15)$ & $0.61(0.13)$ & $0.28(0.13)$ & $1.6(1.5)$ & $3.1(4.1)$ & $0.7(3.7)$ & $2.5(2.4)$ & $-0.7(4.2)$ \\
\hline TAE & $0.46(0.03)$ & $0.36(0.19)$ & $0.56(0.13)$ & $0.59(0.12)$ & $0.31(0.11)$ & $-1.7(1.5)$ & $-5.0(3.9)$ & $0.3(4.5)$ & $2.0(3.1)$ & $-7.4(5)$ \\
\hline VAD & $0.56(0.03)$ & $0.43(0.20)$ & $0.69(0.13)$ & $0.69(0.12)$ & $0.37(0.13)$ & $0.3(1.7)$ & $-5.4(4.6)$ & $4.0(3.9)$ & $0.5(2.4)$ & $-1.9(5.1)$ \\
\hline WAE & $0.46(0.03)$ & $0.41(0.19)$ & $0.58(0.15)$ & $0.53(0.14)$ & $0.33(0.13)$ & $-2.3(1.5)$ & $-6.1(4)$ & $-2.1(4.1)$ & $1.0(3)$ & $-6.3(4.3)$ \\
\hline WYN & $0.44(0.02)$ & $0.29(0.18)$ & $0.55(0.12)$ & $0.60(0.12)$ & $0.30(0.11)$ & $-1.2(1.6)$ & $-5.2(4.3)$ & $1.6(5)$ & $2.6(3.3)$ & $-6.7(4.7)$ \\
\hline
\end{tabular}



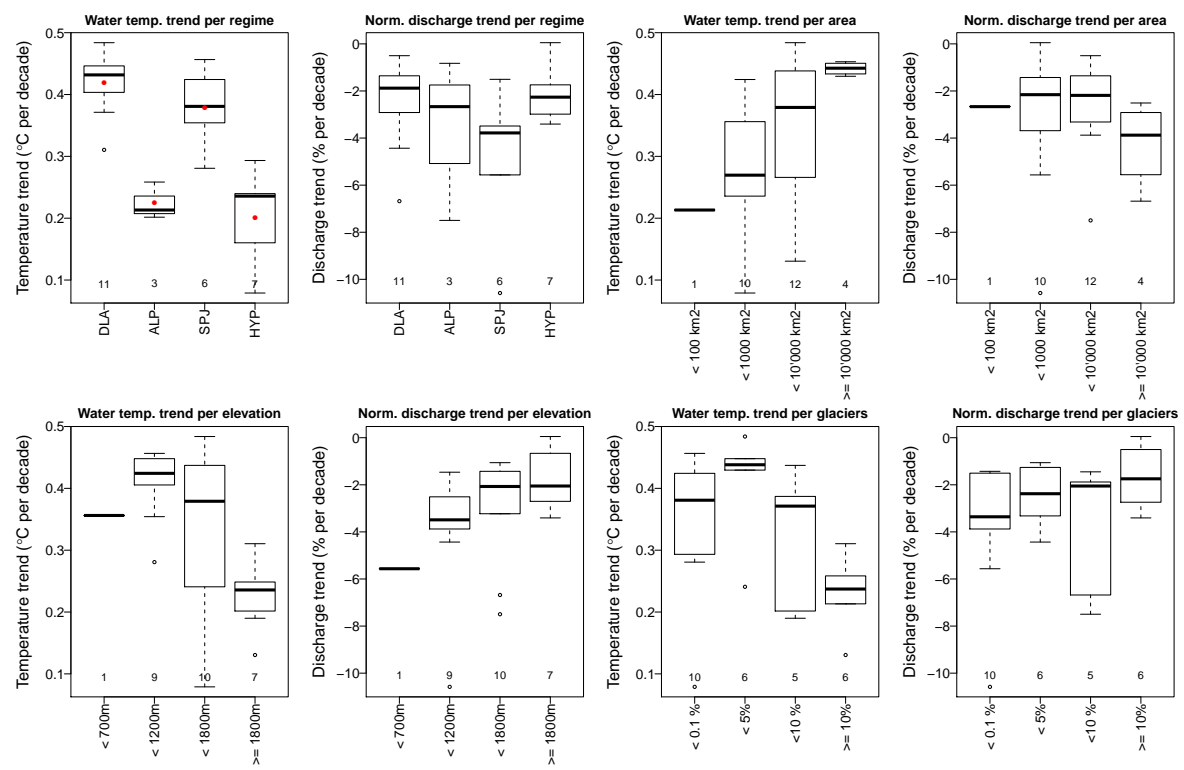

Figure S12. Water temperature and discharge trends for the period 1979-2019 for the 27 catchments where data are available for temperature and discharge (see Table 1 in main text). Top left: classified according to the four different hydrological regimes (DLA = downstream lake regimes, ALP = alpine regimes, SPJ = Swiss Plateau/Jura regimes and HYP = strong influence from hydropeaking). Top right: Classified according to catchment area. Bottom left: Classified according to the catchment elevation. Bottom right: Classified according to the glacier coverage. 

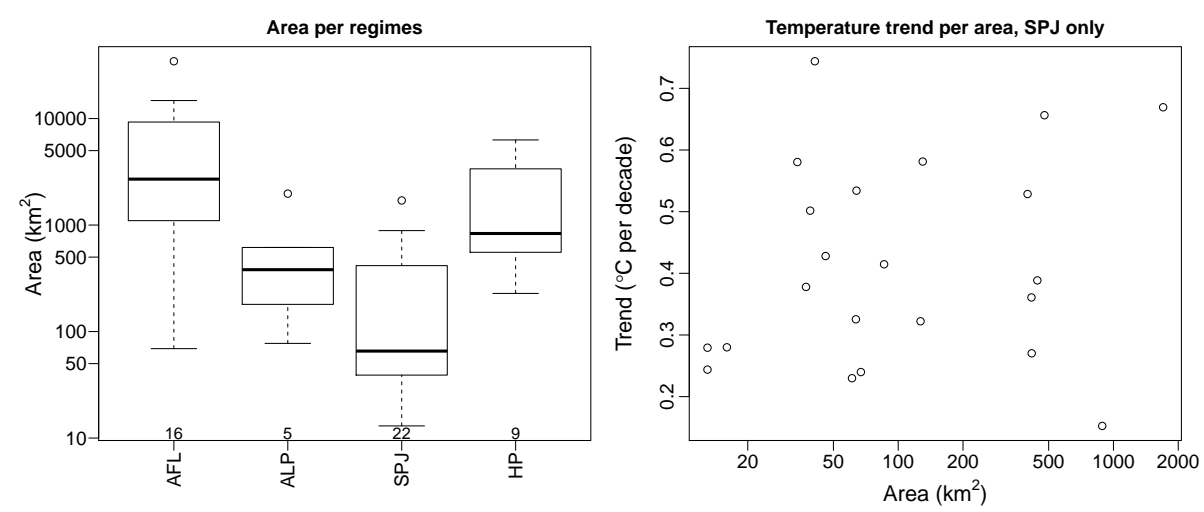

Figure S13. Left: Distribution of catchment area for four different regimes $(\mathrm{DLA}=$ downstream lake regimes, ALP $=$ alpine regimes, SPJ $=$ Swiss Pateau/Jura regimes and HYP = strong influence from hydropeaking). Right: Temperature trends for SPJ regime catchments only. 


\section{S2.2 Lake effect}

This Section presents plots for the four lakes not shown in the main text Section 4.3: Lake Walen (Figure S14), Lake Luzern (Figure S15), Lakes Brienz and Thun (Figure S16), and Lake Biel (Figure S17). The values for the various trends presented are shown in Table 3 in the main text.
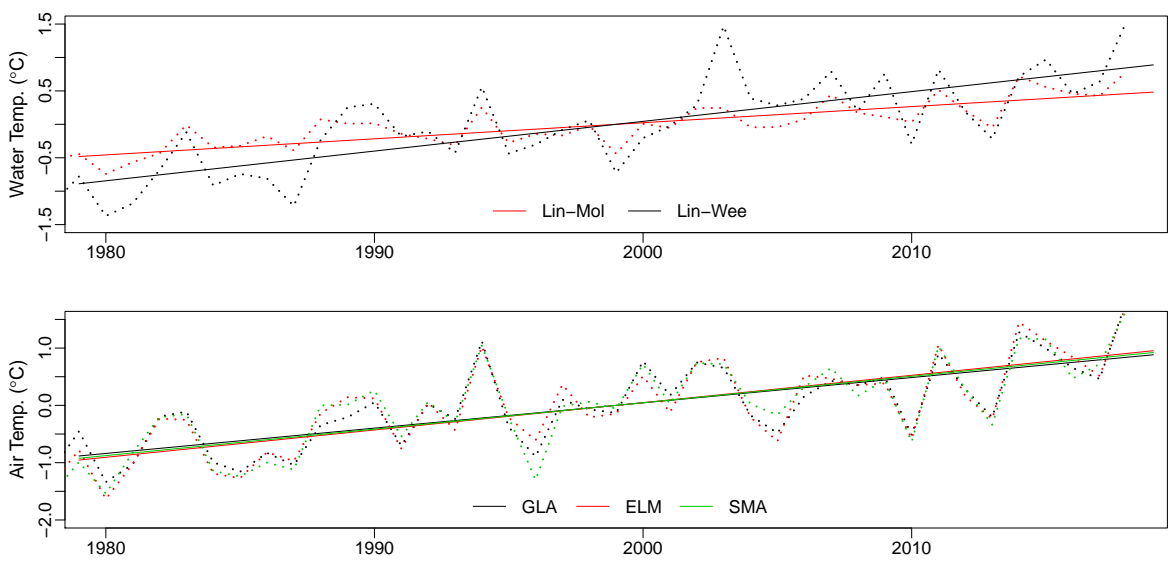

Figure S14. Lake Walen: Water temperature anomalies and trends for inflow and outlet stations (top), air temperature anomalies and trends for surrounding MeteoSwiss stations (bottom). The period for trend computations is 1979-2018. The abbreviation for water gauging stations and for MeteoSwiss stations are given in Table 1 in main text and in Table S2.
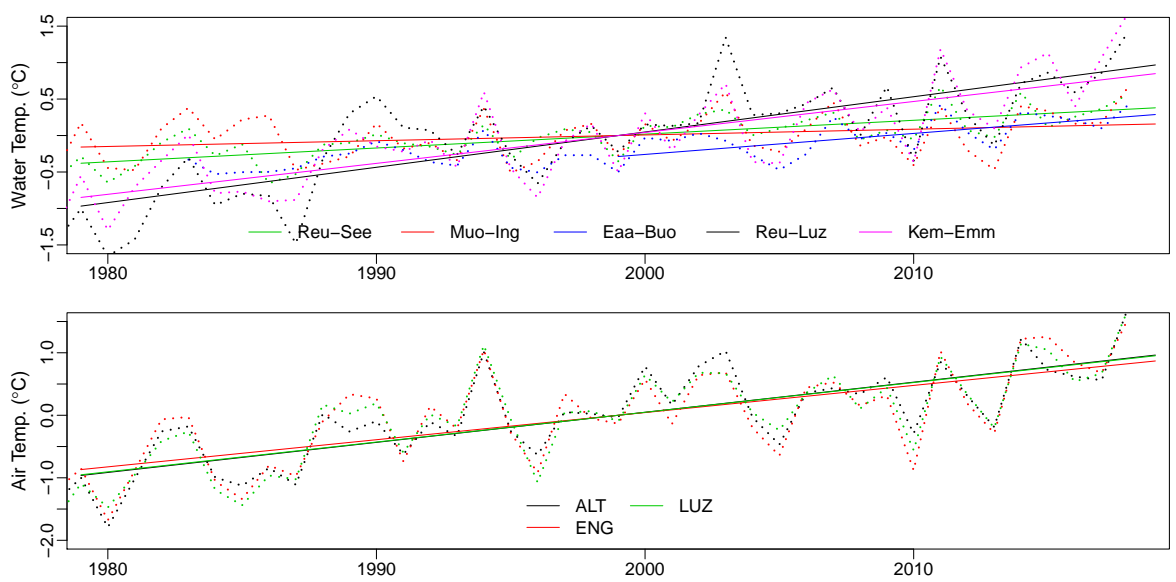

Figure S15. Lake Luzern: Water temperature anomalies and trends for inflow and outlet stations (top), air temperature trends for surrounding MeteoSwiss stations (bottom). The period for trend computations is 1979-2018.The abbreviation for water gauging stations and for MeteoSwiss stations are given in Table 1 in main text and in Table S2. 

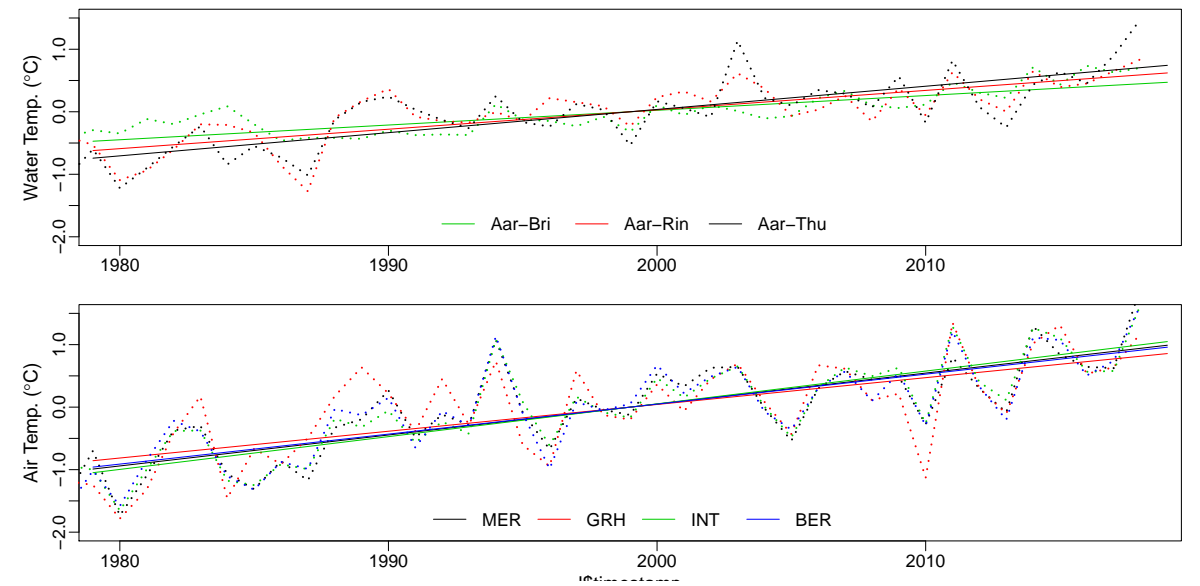

Figure S16. Lakes Brienz and Thun: Water temperature anomalies and trends for inflow and outlet stations (top), air temperature anomalies and trends for surrounding MeteoSwiss stations (bottom). The period for trend computations is 1979-2018. The abbreviation for water gauging stations and for MeteoSwiss stations are given in Table 1 in main text and in Table S2.
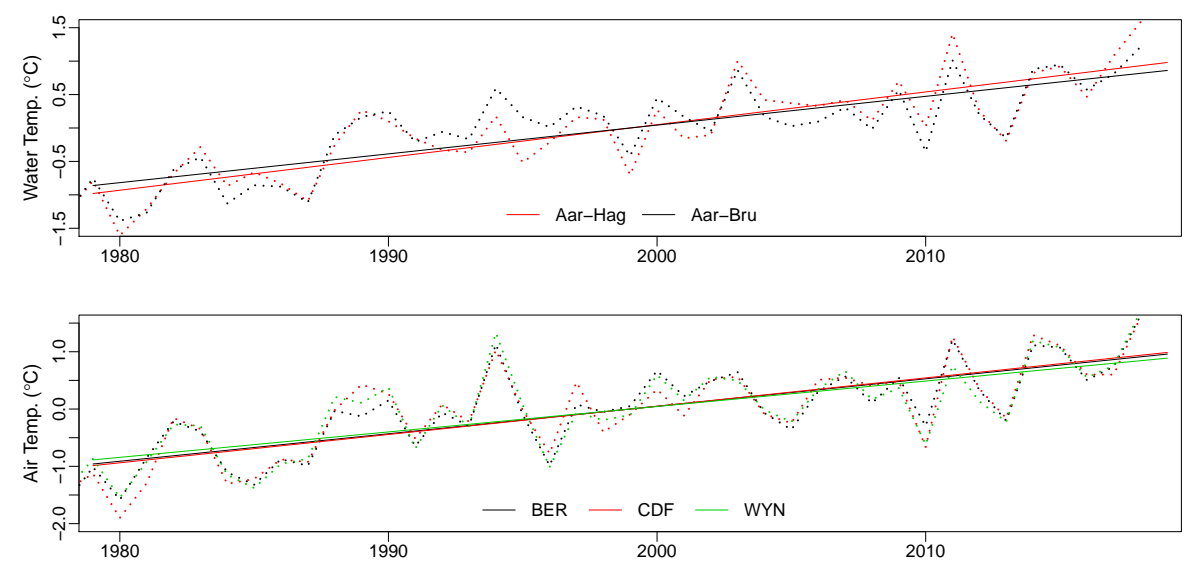

Figure S17. Lake Biel: Water temperature anomalies and trends for inflow and outlet stations (top), air temperature anomalies trends for surrounding MeteoSwiss stations (bottom). The period for trend computations is 1979-2018. The abbreviation for water gauging stations and for MeteoSwiss stations are given in Table 1 in main text and in Table S2. 


\section{S2.3 Seasonal trends and relation with air temperature and precipitation}

This Section presents additional results related to Section 4 of the main text. Figures S18 and S19 show the decades evolution of air temperature and precipitation for the four seasons, similar to Figures 8 and 9 of the main text for stream temperature and discharge.

5 Table S5 shows the correlation between trends of various variables. As discussed in the main text, these correlations are mostly not significant and thus not considered in the study. Therefore, the inter-variable and inter-seasonal mechanisms are used with raw values instead of trends.

Figures S20 and S22 show the yearly anomalies in stream temperature, discharge, air temperature and precipitation in winter and fall, similar to Figures 8 and 11 in the main text which present spring and summer.

Finally, Figure S21 shows the snow water equivalent (SWE) at the beginning of various months over the whole country, and Figure S23 shows the evolution of the summer mass balance for 9 Swiss glaciers.
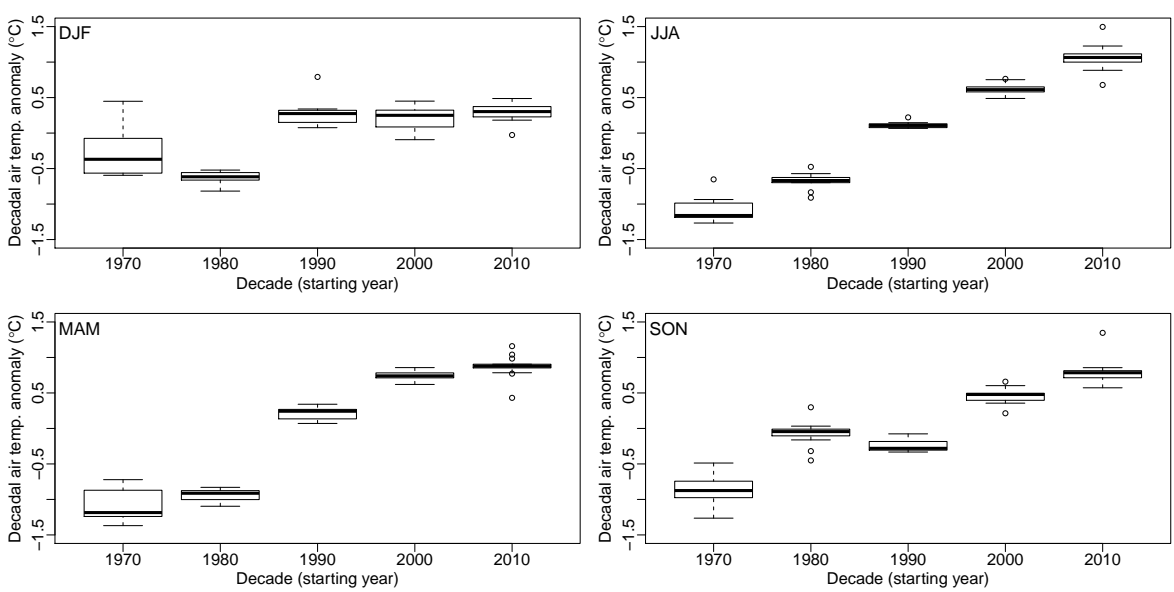

Figure S18. Air temperature seasonal anomalies over the 14 catchments where data are available since 1970 (see Table S2). Anomalies with respect to the 1970-2018 period. 

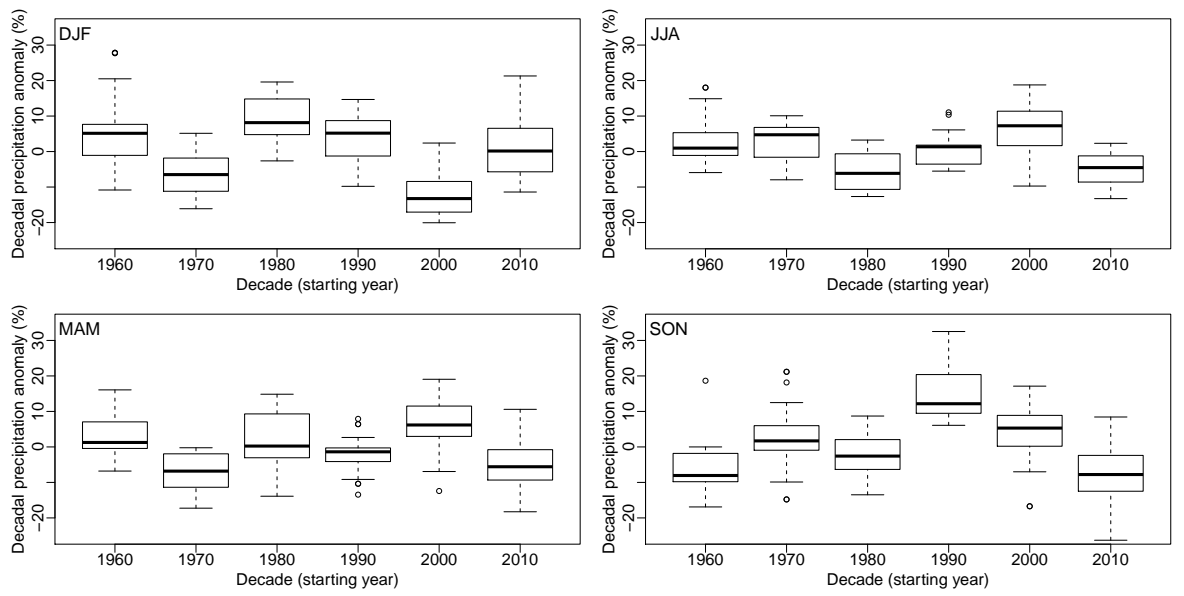

Figure S19. Precipitation seasonal relative anomalies over the 26 stations where data are available since 1960 (see Table S2). Anomalies with respect to $1960-2018$ period.

Table S5. Correlation between the trends of water and air temperature (left), water temperature and discharge (middle) and discharge and precipitation (right). Correlations are computed between annual and seasonal trends, and by taking one value per catchment and constructing ordered vectors of values. The number in parenthesis indicates the p-value of the null-hypothesis (no correlation). Since the computation here is different to the one in Table 4 in main text (where correlation is computed from full time series and then averaged between catchment), the two tables cannot be compared.

\begin{tabular}{|c|c|c|c|c|c|}
\hline \multicolumn{2}{|c|}{$\begin{array}{c}\text { Water and air } \\
\text { temperature trends }\end{array}$} & \multicolumn{2}{|c|}{$\begin{array}{l}\text { Water temperature } \\
\text { and discharge trends }\end{array}$} & \multicolumn{2}{|c|}{$\begin{array}{c}\text { Discharge and } \\
\text { precipitation trends }\end{array}$} \\
\hline Period & cor. & Period & Cor. & Period & Cor. \\
\hline Yearly & $-0.18(0.19)$ & Yearly & $-0.25(0.08)$ & yearly & $0.08(0.01)$ \\
\hline Winter & $-0.13(0.36)$ & Winter & $-0.50(<0.01)$ & Winter & $0.36(0.02)$ \\
\hline Spring & $0.02(0.87)$ & Spring & $-0.35(0.01)$ & Spring & $0.11(0.43)$ \\
\hline Summer & $-0.09(0.51)$ & Summer & $-0.05(0.72)$ & Summer & $0.33(0.01)$ \\
\hline Fall & $-0.26(0.07)$ & Fall & $-0.26(0.06)$ & Fall & $0.34(0.58)$ \\
\hline
\end{tabular}



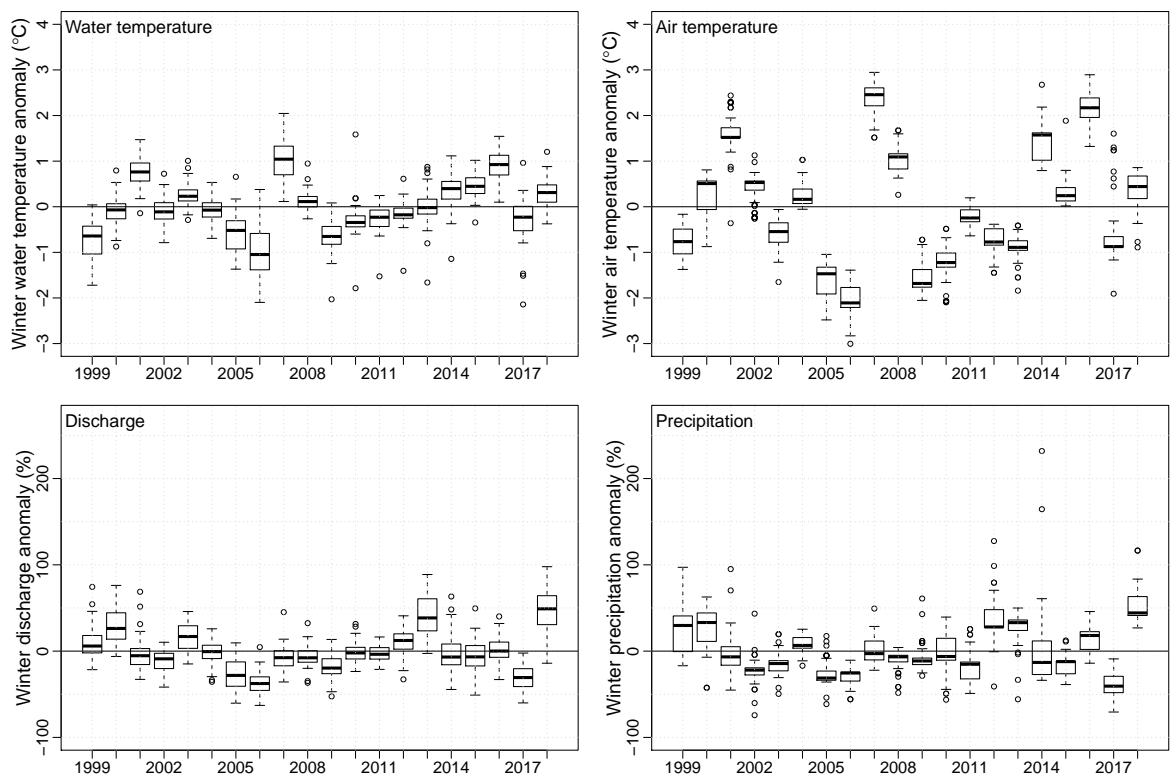

Figure S20. Winter anomalies in stream temperature, air temperature, relative discharge and relative precipitation for all catchments. Anomalies are computed with respect to the 1999-2018 mean for each catchment.

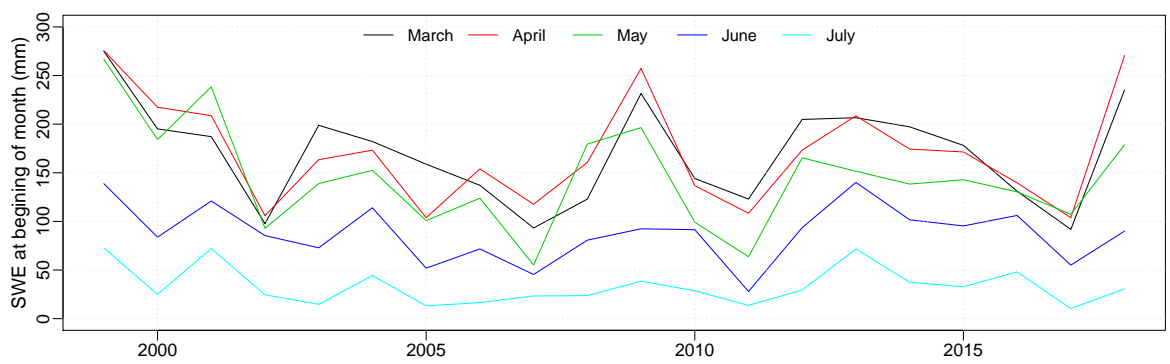

Figure S21. Snow water equivalent in spring in Switzerland at the beginning of the months, from March to July. Obtained from Magnusson et al. (2014) and provided by the WSL Institute for Snow and Avalanche Research (SLF). 

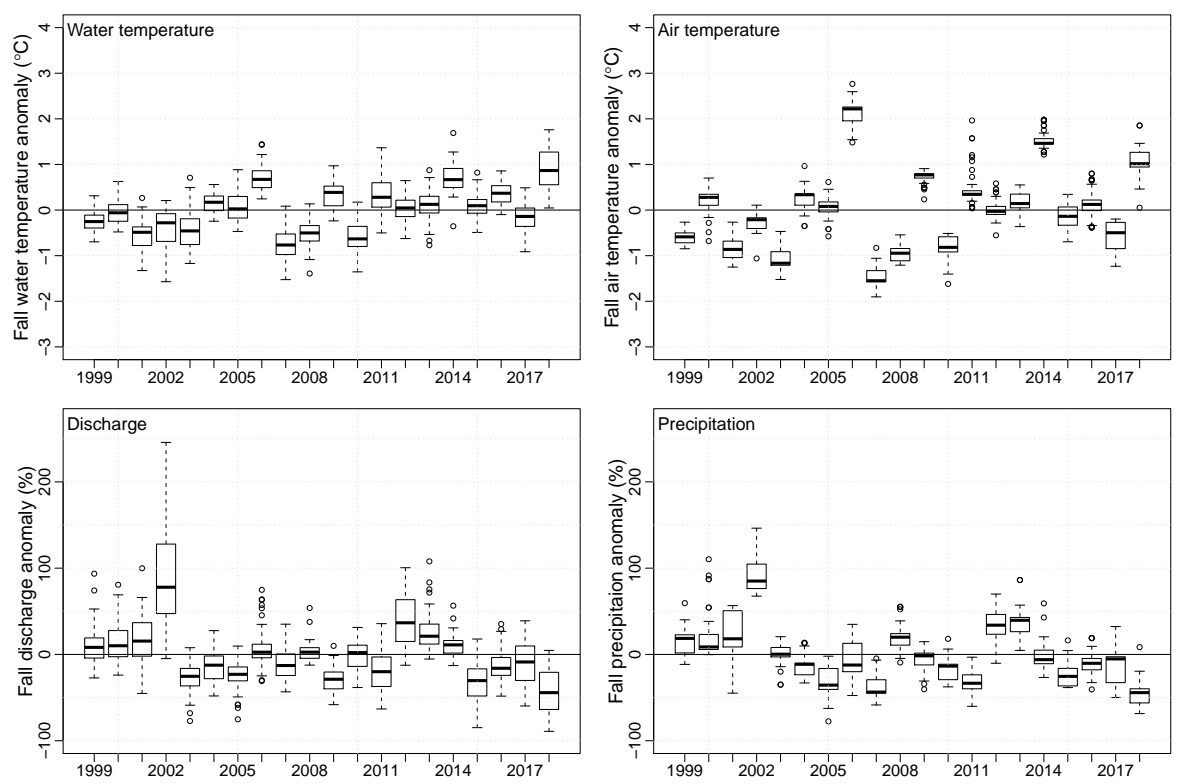

Figure S22. Fall anomalies in stream temperature, air temperature, relative discharge and relative precipitation for all catchments. Anomalies are computed with respect to the 1999-2018 mean for each catchment.

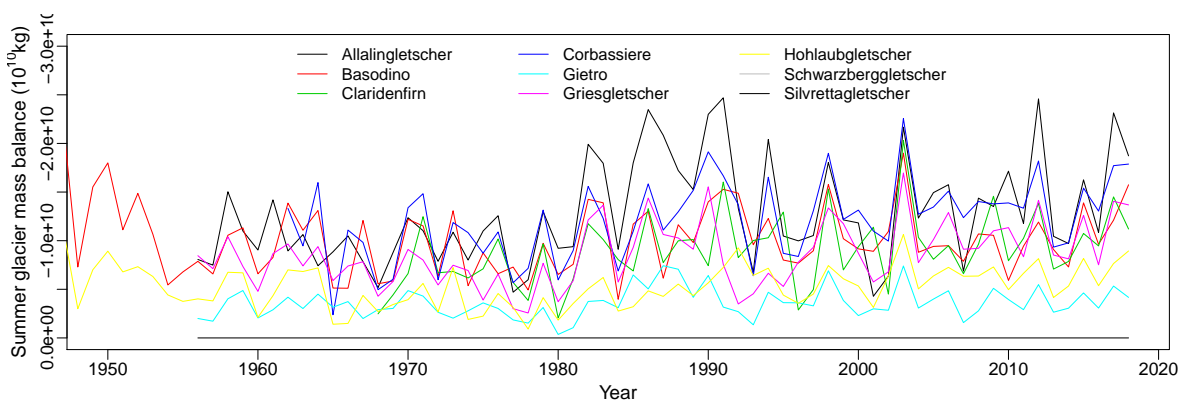

Figure S23. Summer mass balance for 9 Swiss glaciers, from (GLAMOS, 2018). 


\section{S2.4 Ecological indicators}

This Section presents an additional Figure related to Section 4.5 of the main text. Figure S24 shows the summer runoff anomaly for the same catchments as the ones used in Figure 17 in the main text.

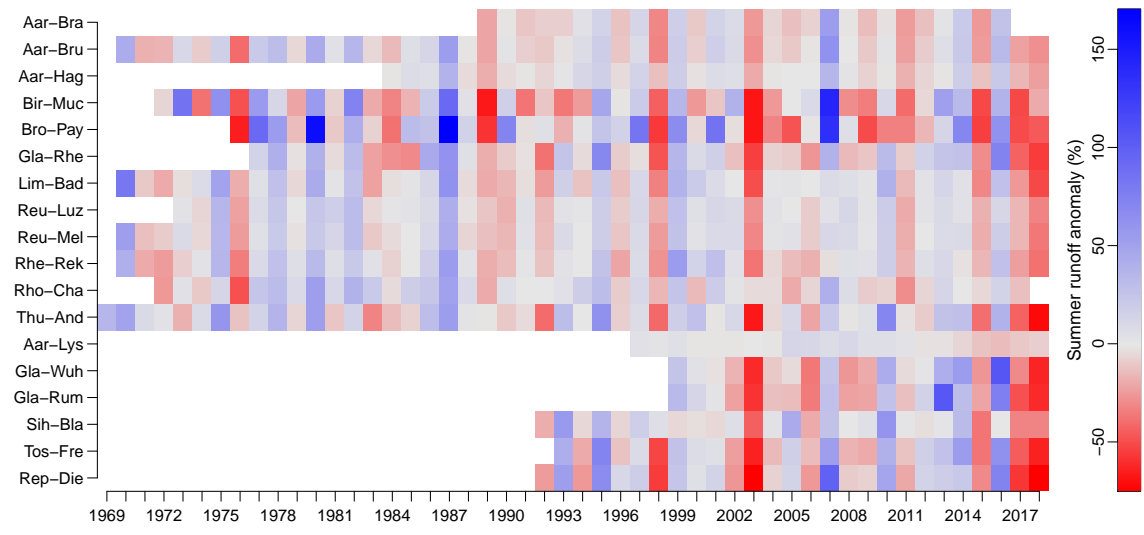

Figure S24. Summer relative runoff anomaly (with respect to the $1999-2018$ period) for the catchments in which the $25^{\circ} \mathrm{C}$ threshold is reached (see Figure 17 in main text).

\section{Code and data availability. TEXT}

The aim is to share the data set collected in the context of this paper, discussions are still going on with data providers to find an agreement. If successful, the dataset will be made available on EnviDat.ch.

The code will also be available in a well commented and comprehensive form at publication time.

For the time being (review) documented code and data are available at: https://drive.switch.ch/index.php/s/9BEqpBWFkNWoTSU The main author should be contacted to obtain the password (adrien.michel@epfl.ch). 


\section{References}

Enfield, D., Mestas-Nunez, A., and Trimble, P.: The Atlantic multidecadal oscillation and its relation to rainfall and river flows in the continental US, Geophysical Research Letters, 28, 2077-2080, 2001.

GLAMOS: Swiss Glacier Mass Balance, release 2018, Glacier Monitoring Switzerland, doi:10.18750/massbalance.2018.r2018, 2018.

5 Jones, P. D., Jonsson, T., and Wheeler, D.: Extension to the North Atlantic oscillation using early instrumental pressure observations from Gibraltar and south-west Iceland, International Journal of Climatology, 17, 1433-1450, https://doi.org/10.1002/(SICI)10970088(19971115)17:13<1433::AID-JOC203>3.0.CO;2-P, $\quad$ https://rmets.onlinelibrary.wiley.com/doi/abs/10.1002/\%28SICI\% 291097-0088\%2819971115\%2917\%3A13\%3C1433\%3A\%3AAID-JOC203\%3E3.0.CO\%3B2-P, 1997.

Lehre Seip, K., Gron, O., and Wang, H.: The North Atlantic oscillations: Cycle times for the NAO, the AMO and the AMOC, Climate, 7, 43, 10 https://doi.org/10.3390/cli7030043, 2019.

Magnusson, J., Gustafsson, D., Hüsler, F., and Jonas, T.: Assimilation of point SWE data into a distributed snow cover model comparing two contrasting methods, Water Resources Research, 50, 7816-7835, https://doi.org/10.1002/2014WR015302, https://agupubs.onlinelibrary. wiley.com/doi/abs/10.1002/2014WR015302, 2014. 